Please do not remove this page

RMIT

UNIVERSITY

\title{
Nonequilibrium molecular dynamics simulation of dendrimers and hyperbranched polymer melts undergoing planar elongational flow
}

Hajizadeh, Elnaz; Todd, Billy; Daivis, Peter

https://researchrepository.rmit.edu.au/esploro/outputs/9921859455401341/filesAndLinks?institution=61RMIT_INST\&index=null

Hajizadeh, E., Todd, B., \& Daivis, P. (2014). Nonequilibrium molecular dynamics simulation of dendrimers and hyperbranched polymer melts undergoing planar elongational flow. Journal of Rheology, 58(2), 281-305. https://doi.org/10.1122/1.4860355

Document Version: Published Version

Published Version: https://doi.org/10.1122/1.4860355

Repository homepage: https://researchrepository.rmit.edu.au

(C) 2014 The Society of Rheology

Downloaded On 2023/04/26 22:48:47 +1000 
Thank you for downloading this document from the RMIT Research Repository.

The RMIT Research Repository is an open access database showcasing the research outputs of RMIT University researchers.

RMIT Research Repository: http://researchbank.rmit.edu.au/

\section{Citation:}

Hajizadeh, E, Todd, B and Daivis, P 2014, 'Nonequilibrium molecular dynamics simulation of dendrimers and hyperbranched polymer melts undergoing planar elongational flow', Journal of Rheology, vol. 58, no. 2, pp. 281-305.

See this record in the RMIT Research Repository at:

http://researchbank.rmit.edu.au/view/rmit:24640

Version: Published Version

Copyright Statement: (c) 2014 The Society of Rheology

Link to Published Version:

http://dx.doi.org/10.1122/1.4860355 
Copyright 2014 The Society of Rheology. This article may be downloaded for personal use only. Any other use requires prior permission of the author and the American Institute of Physics.

The following article appeared in Journal of Rheology, vol. 58, no. 2, March/April 2014, pp. 281-305, and may be found at http://dx.doi.org/10.1122/1.4860355 .

The Society of Rheology is a member of the American Institute of Physics (AIP). 


\title{
Nonequilibrium molecular dynamics simulation of dendrimers and hyperbranched polymer melts undergoing planar elongational flow
}

\author{
Elnaz Hajizadeh ${ }^{\text {a) }}$ and B. D. Todd ${ }^{\text {) }}$ \\ Department of Mathematics, Faculty of Science, Engineering and Technology, \\ and Centre for Molecular Simulation, Swinburne University of Technology, \\ Hawthorn, Victoria 3122, Australia
}

P. J. Daivis ${ }^{\text {c) }}$

School of Applied Sciences, RMIT University, Melbourne, Victoria 3001, Australia

(Received 11 August 2013; final revision received 15 December 2013;

published 10 January 2014)

\begin{abstract}
Synopsis
The planar elongational melt rheology and structural properties of dendrimers and hyperbranched polymers of different molecular weights (generations 1-4) and their linear counterparts have been studied using nonequilibrium molecular dynamics simulation techniques in the isothermal-isobaric ensemble. The extensional viscosity showed three distinctive regions against strain-rate, including an initial Newtonian region at low strain-rates, followed by a thickening behavior at medium strain-rates and terminated with a thinning region at very high strain-rates, in agreement with the Sarkar and Gupta model [J. Rein. Plast. Comp. 20, 1473-1484 (2001)]. In addition, a structural analysis was performed to study the size, shape, and spatial distributions within globular dendrimers and hyperbranched polymer molecules under planar elongational flow (PEF). Ratios of the eigenvalues of the gyration tensor showed that contrary to shear flow, under PEF even at low strain rates, dendrimers and hyperbranched molecules have ellipsoidal conformations and change to a much more flattened prolate shape at higher strain rates. In combination with the eigenvalue ratios, the distribution of monomers from the central core of the molecules showed that the thickening region occurs due to branches being stretched, and terminal thinning behavior stems primarily from flow-induced alignment and finite extensibility effects. (C) 2014 The Society of Rheology. [http://dx.doi.org/10.1122/1.4860355]
\end{abstract}

\section{INTRODUCTION}

Perhaps one of the most significant challenges for rheologists is the determination of planar extensional viscosity, whether by experimental methods or simulation techniques. This is reflected in the very small number of experimental and simulation studies available

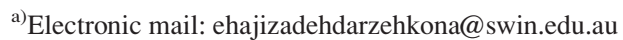

b) Author to whom correspondence should be address; electronic mail: btodd@swin.edu.au

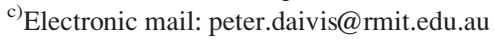


in the literature, which is due to the difficulties associated with generation and control of extensional flows, as has been reviewed by Dealy (1971), Petrie (2006), and McKinley and Sridhar (2002). Extensional flows are important for industry because of their application in fiber spinning and film forming processes [Weinbergert and Goddard (1974)] and also for academia, as a guide for formulating constitutive equations for the rheology of non-Newtonian fluids [Tanner and Nasseri (2003); McLeish and Larson (1998); Ilg and Kröger (2011)]. As most constitutive equations are tube-based models, they are not applicable to systems with molecular weights under the entanglement molecular weight.

Dendritic polymers, including dendrimers and random hyperbranched polymers, are synthetic treelike molecules that have received significant interest over the past 20 years due to their unusual properties compared to conventional linear polymers [Tomalia et al. (1984)]. Since the introduction of this new class of materials, many papers [such as those by Flory (1941); Kim and Webster (1990); Frechet et al. (1994); Wooley et al. (1991); Nunez et al. (2000); Hsieh et al. (2001)] have been published on the synthesis and characterization of different generations of hyperbranched polymers and dendrimers, revealing unique macroscopic properties of these polymers compared to their linear counterparts, such as low melt viscosity, due to their compact structure. Recently, Silva et al. (2012) introduced dendrimers as a potential platform in nanotechnology-based drug delivery systems due to their specific physical and chemical properties arising from their organized architecture.

The shape and the orientation of macromolecules in polymer melts dictate their dynamical behavior and rheological properties. Understanding these structural effects is crucial in increasing our understanding of the relationship between microstructure and macroscopic properties of this class of materials. Experimental techniques such as neutron scattering [Frick and Richter (1995)], neutron spin-echo (NSE) [Likhtman (2005)], dielectric spectroscopy, and NMR have been very influential in illustrating the structure and dynamics of polymer melts, but there are properties that these experimental techniques fail to probe. For example, in the case of branched polymers, experimental techniques such as NMR are useful in determining the number of branches. However, in measuring the length of branches, NMR cannot differentiate branches that have more than six-ten atoms [Gaborieau and Castignolles (2011)]. However, molecular simulation techniques, such as molecular dynamics, offer an alternative approach.

There are also significant advances in molecular dynamics simulations which can potentially directly calculate macroscopic properties from the microscopic properties of a polymer melt. For this reason, computer simulations, especially nonequilibrium molecular dynamics (NEMD) simulation, can play an important role in our understanding of polymer melt behavior under different flow conditions. The advantage of computer simulation over experimental methods is that complex architectures can be simulated with controlled molecular weight and molecular topology. However, the idealized molecules examined by these methods are mostly much shorter than real polymers. Normally, a limited number of molecules are studied in the simulation because of the large number of calculations entailed. Moreover, for larger molecules, the relaxation time is very large and beyond the feasible simulation time. This implies that the molecular weight of the simulated molecules is usually below the entanglement molecular weight. Despite the fact that the molecules in these simulations are short, they can still capture much of the behavior of real polymer melts. For instance, these model molecules often reproduce nonlinear behavior and normal stress differences that are observed for real polymer melts. NEMD simulations have shown [Matin et al. (2003b)] that liquids that often appear Newtonian in experiments at low strain rates show non-Newtonian behavior if they are deformed at large enough strain rates, as in NEMD simulations. Furthermore, these simulations can also be used to capture the effect of molecular shape on the rheological 
behavior. Understanding of these structural effects can boost our general knowledge and provides guidance in devising theoretical models for real polymers.

Although numerous computational studies have been performed to investigate the dynamics of polymeric fluids [for instance, van Ruymbeke et al. (2006); Karayiannis and Mavrantzas (2005); Hess (2002); Hoover and Hoover (2003)], only a few of these simulation studies focus on melt rheology [e.g., works by van Ruymbeke et al. (2007); Wagner and Rolon-Garrido (2009); Likhtman et al. (2007); Daivis et al. (1992); Laun and Münstedt (1978); Aust et al. (1999)] which, among them, elongational behavior studies are very limited. For example, for molecular dynamics simulations, see works by Heyes (1985), Hounkonnou et al. (1992), Todd and Daivis (1998), Kalra and Joo (2009), and Baranyai and Cummings (1999). Also, several authors conducted Brownian dynamics simulations in order to study different types of extensional flow behavior of polymeric solutions, such as Cifre and de la Torre (1999), Sridhar et al. (2007), and Neelov and Adolf (2003, 2004).

Matin et al. (2003b) and Daivis et al. (2007) successfully applied NEMD techniques to study melt rheological behavior of linear polymers. Le et al. (2009a, 2009b) and Bosko et al. (2004a, 2004b) studied melt rheology and structural behavior of hyperbranched polymers and dendrimers under shear flow, respectively, but to the best of our knowledge, this current paper is the first NEMD study, coupled with the Kraynik-Reinelt (1992) (KR) boundary condition method, to study the melt rheology of dendrimers and hyperbranched polymer melts and their shape and orientational changes under planar elongational flow (PEF). Our aim here is to investigate the effect of microscopic structural changes and flowinduced molecular deformation under PEF on the observed macroscopic properties such as extensional viscosity and obtain greater physical intuition into the microscopic reasons behind the macroscopic properties observed for dendrimers and hyperbranched molecules under such flow conditions. This study can elucidate the effect of highly branched and globular architecture on nonlinear viscoelastic properties of this new class of material.

The Carreau-Yasuda model has been used over the past decades to model the strainrate dependent steady shear viscosity for polymeric melts. We refer the reader to the book by Bird et al. (1987) for details. Sarkar and Gupta (2001) modified this model for the elongational melt viscosity by incorporating one extra term for the thickening phenomenon which is observed in the elongational behavior of polymer melts. We use the Sarkar-Gupta model in our studies and calculate the adjustable parameters of the model by fitting them to our simulation data. The longest relaxation times were further approximated for the systems under study.

The remainder of this paper is organized as follows. Section II A describes the molecular model we used to design our polymers. Section II B presents the isothermal-isobaric (NPT)NEMD algorithm. In Sec. III A, we present the results and a discussion of the rheological behavior of dendrimers and hyperbranched polymers, compared to their linear counterparts undergoing both PEF and shear flow. We also discuss the application of the Sarkar and Gupta model for the strain rate dependent elongational viscosity. Section III B focuses on the structural properties of the systems under study such as gyration tensor analysis, mass distribution within the molecules and its variations during PEF and shear flow, and also the distribution of terminal groups within the molecule. Section IV presents the conclusion.

\section{METHODOLOGY}

\section{A. Dendrimer and hyperbranched model}

Dendrimers and hyperbranched polymers were simulated using a coarse-grained uniform bead model. These basic units correspond to the linear units or branching 
points of the molecule and are interconnected to create treelike structures. Beads along the chain can rotate and vibrate freely. All beads (monomers) are identical and indistinguishable except for their position in the molecule. The total number of monomers in a dendrimer can be defined as $N=f b\left((f-1)^{g+1}-1\right) /(f-2)$, where $f$ is the functionality of end groups, $b$ is the number of monomers in the chain units, and $g$ is the generation number. With the choice of $f=3$ and $b=2$, dendrimers of generations 1,2 , 3 , and 4 will have 19, 43, 91, and 187 monomers, respectively. In order to compare our simulation data for hyperbranched polymers with those of dendrimers, a simple specific architecture of hyperbranched polymers has been chosen. They are dendrimers with trifunctional end groups $f=3$ and two monomers in the chain units $b=2$ that have one imperfect branching point $f=2$. As our hyperbranched polymers have the same number of monomers as the dendrimers but fewer branches at one branching point, extra monomers are added in the outermost layer of the molecules with $f=3$ and $b=2$ (see Fig. 1). Models of linear polymers of length 19, 43, 91, and 187 have been built by successively attaching the same monomers. More details can be found in Le et al. (2009a) and Bosko et al. (2004a). In this model, only bond and pairwise interactions are taken into account. Monomers comprising the melt interact via the WeeksChandler-Anderson (WCA) potential, which is a shifted and truncated Lennard-Jones (LJ) potential, and also a finitely extensible nonlinear elastic (FENE) potential [details can be found in papers by Kröger and Hess (2000) and also Kremer and Grest (1990)]. The WCA potential is given as

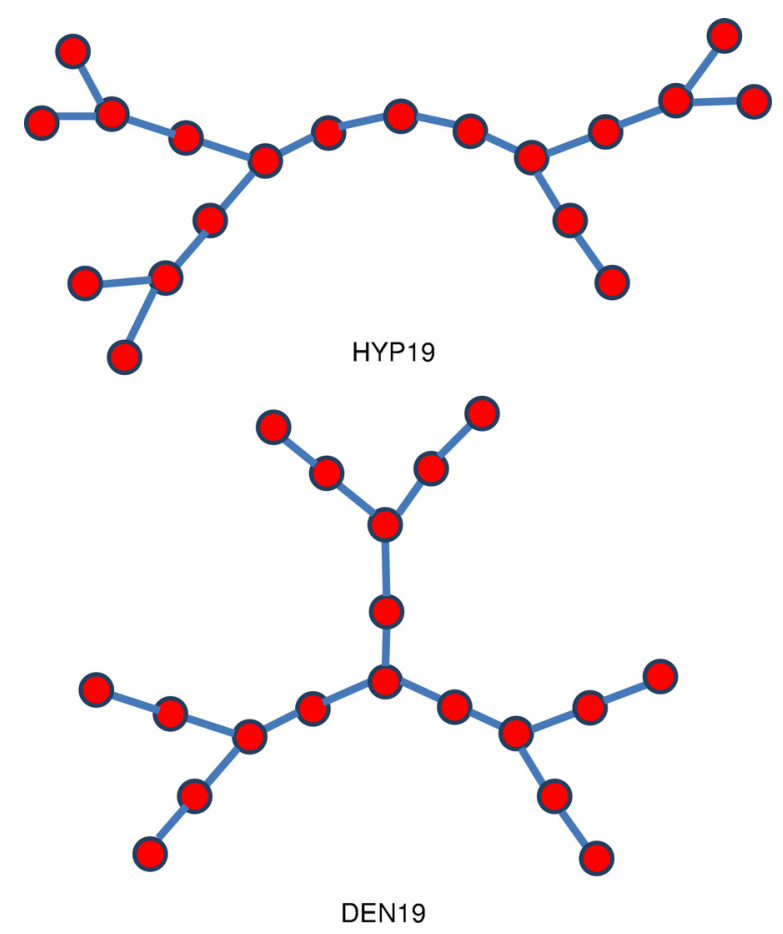

FIG. 1. Schematic representation for model hyperbranched and dendrimer molecules of generation 1 (19 beads per molecule), revealing functionality, and length of the spacing of both hyperbranched and dendrimer molecules $(f=3$ and $b=2)$, and position of the missing branch for hyperbranched molecule. 


$$
U_{i j}^{W C A}= \begin{cases}4 \epsilon\left[\left(\frac{\sigma}{r_{i j}}\right)^{12}-\left(\frac{\sigma}{r_{i j}}\right)^{6}\right]+\epsilon & \text { for } \quad \frac{r_{i j}}{\sigma}<2^{1 / 6} \\ 0 & \text { for } \quad \frac{r_{i j}}{\sigma} \geq 2^{1 / 6}\end{cases}
$$

where $r_{i j}$ is the separation between the sites represented by monomers $i$ and $j, \epsilon$ is the potential well depth, and $\sigma$ is the effective diameter of the monomers. This potential results in a purely repulsive force that includes the effect of excluded volume. The FENE potential is expressed as

$$
U_{i j}^{F E N E}= \begin{cases}-0.5 k R_{0}^{2} \ln \left[1-\left(r_{i j}\right) / R_{0}^{2}\right] & \text { for } \quad r_{i j} \leq R_{0} \\ \infty & \text { for } \quad r_{i j} \geq R_{0}\end{cases}
$$

where $R_{0}$ is a finite extensibility and $k$ is a spring constant. In this work, $R_{0}$ and $k$ were set to 1.5 and 30, respectively, as is typical [Kröger and Hess (2000); Kröger (2004); Kremer and Grest (1990)]. For this choice of parameters, the maximal extent of bonds is short enough to prevent crossing of branches, whereas the magnitude of the bonding force is small enough to enable simulations with relatively large time steps. Nonbonded monomers only have WCA potential interactions, whereas bonded monomers have both FENE and WCA interactions which create a potential well for the flexible bonds that maintains the architecture of the molecules. In the remainder of this paper, all quantities are expressed in terms of site reduced units in which the reduction parameters are the LJ interaction parameters $\epsilon$ and $\sigma$ and the mass $m_{i \alpha}$ of bead $\alpha$ in molecule $i$. The reduced temperature is given by $T^{*}=k_{B} T / \epsilon$, the density by $\rho^{*}=\rho \sigma^{3}$, the pressure tensor by $\mathbf{P}^{*}=\mathbf{P} \sigma^{3} / \epsilon$, and strain rate by $\dot{\gamma}^{*}=\dot{\gamma}\left(m \sigma^{2} / \epsilon\right)^{1 / 2}$. For simplicity of notation, the asterisk will be omitted hereafter. In all simulations we set $\sigma=\epsilon=m_{i \alpha}=k_{B}=1$.

\section{B. NEMD simulation}

Our NEMD simulations are based on the molecular version of the homogeneous NPT SLLOD equations of motion [details can be found in Todd and Daivis (2007) and Frascoli and Todd (2007)]

$$
\begin{gathered}
\dot{\mathbf{r}}_{i \alpha}=\frac{\mathbf{p}_{i \alpha}}{m_{i \alpha}}+\dot{\epsilon}\left(\mathbf{i} x_{i \alpha}-\mathbf{j} y_{i \alpha}\right)+\dot{\zeta} \frac{m_{i \alpha}}{M_{i}} \mathbf{r}_{i}, \\
\dot{\mathbf{p}}_{i \alpha}=\mathbf{F}_{i \alpha}-\dot{\epsilon}\left(\mathbf{i} p_{x i \alpha}-\mathbf{j} p_{y i \alpha}\right)-\alpha \frac{m_{i \alpha}}{M_{i}} \mathbf{p}_{i}-\frac{m_{i \alpha}}{M_{i}} \dot{\zeta} \mathbf{p}_{i},
\end{gathered}
$$

where $\mathbf{r}_{i \alpha}$ and $\mathbf{p}_{i \alpha}$ represent the position and thermal momentum of monomer $\alpha$ on molecule $i, \dot{\epsilon}$ is the elongational strain rate, $\mathbf{p}_{i}$ is the momentum of the molecular center of mass of molecule $i$, and $M_{i}$ is the mass of molecule $i$. Our geometry is such that expansion occurs in the $x$-direction, whereas contraction occurs in the $y$-direction. The simulations were performed at constant temperature using a molecular version of the Gaussian thermostat with a constraint multiplier $\alpha$ given by

$$
\alpha=\frac{\sum_{i=1}^{N}\left(\mathbf{F}_{i} \cdot \mathbf{p}_{\mathbf{i}}-\dot{\epsilon}\left(p_{x i}^{2}-p_{y i}^{2}\right)\right) / M_{i}}{\sum_{i=1}^{N} \mathbf{p}_{i}^{2} / M_{i}}-\dot{\zeta}
$$


Constant pressure simulations were accomplished by coupling the system to an extended degree of freedom $\dot{\zeta}$ associated with the volume of the simulation box. Thus, the volume, rather than the pressure, is allowed to fluctuate. The Nosé-Hoover integral feedback mechanism has been implemented in this work [Daivis and Evans (1994)]. The time evolution of $V$ is regulated by the variable $\dot{\zeta}$ via the following equation:

$$
\dot{V}=3 \dot{\zeta} V
$$

where $\dot{V}$ is the first derivative of the cell volume with respect to time. To maintain the target pressure, the lattice vectors have to be rescaled such that the volume of the unit cell is compatible with the equations of motion. This process has been done at each time step and a consideration of the lattice cell evolution for PEF is necessary each time the rescaling process takes place. The dilation rate is obtained by solving the additional differential equation

$$
\ddot{\zeta}=\frac{\left(p-p_{0}\right) V}{Q_{p} N k_{B} T},
$$

where $Q_{p}$ is a damping factor for which the optimal value will depend on the type of system studied, $p$ is the instantaneous isotropic pressure $p=\frac{1}{3} \operatorname{Tr}\left(\mathbf{P}^{M}\right)$, and $p_{0}$ is the target pressure.

The molecular pressure tensor is computed as

$$
\mathbf{P}^{M}=\frac{1}{V}\left\langle\sum_{i=1}^{N_{m}} \frac{\mathbf{p}_{i} \mathbf{p}_{i}}{M_{i}}-\frac{1}{2} \sum_{i=1}^{N_{m}} \sum_{\alpha=1}^{N} \sum_{j \neq i}^{N_{m}} \sum_{\beta=1}^{N} \mathbf{r}_{i j} \mathbf{F}_{i \alpha j \beta}\right\rangle,
$$

where $\mathbf{r}_{i j}=\mathbf{r}_{j}-\mathbf{r}_{i}, \mathbf{r}_{i}$ and $\mathbf{r}_{j}$ are the centers of mass of molecules $i$ and $j$, and $\mathbf{F}_{i \alpha j \beta}$ is the intermolecular force on site $i \alpha$ due to site $j \beta$. Thus, there are a total of $6 N+2$ differential equations to solve: $3 \mathrm{~N}$ for the monomer positions, $3 \mathrm{~N}$ for the peculiar momenta, one for the system volume, and one for the dilation rate. The details of our implementation of the KR periodic boundary conditions for PEF simulations as well as the description of the molecular pressure tensor are given elsewhere [Todd and Daivis $(1998,2000,2007)]$.

The equations of motion of all monomers were integrated using a fifth-order Gear predictor corrector differential equation solver with reduced time step $\Delta t=0.001$. After dendrimer, linear, and hyperbranched polymer systems of 125 molecules generated at low density had been compressed to the required density of $\rho=0.84$, they were equilibrated for typically several million time steps, and the pressure was plotted against time to check if the system had reached the steady state. Ensemble averages of all properties of interest were then calculated by averaging over typically 30 NEMD steady-state trajectories. The temperature was fixed at 1 (reduced unit) for all systems studied.

There are two technical limitations in our NEMD simulations. One occurs at very low strain rates and the other at very high strain rates, especially for high molecular weight systems. The first limitation results in poor statistics for the properties calculated at very low strain rates. To obtain better statistics, one needs to run the simulations for very long times, which is not practical from a computational point of view. At extremely high strain rates, our algorithm fails. This comes from the fact that at very high strain rates, bonds between the monomers are fluctuating very fast and the integration time step is larger than the inverse rate of these fluctuations. As a result, the equations of motion become 
stiff. In order to overcome this limitation, one should reduce the integration time step, which again will demand a higher amount of computational time consumption. Thus in the end, a compromise must be made between the competing demands of strain rate range and computational time accessible.

\section{RESULTS AND DISCUSSION}

\section{A. Rheology}

The rheological properties of molecular melts are typically analyzed using standard material functions. To characterize the melt rheological properties of dendrimers, hyperbranched polymers of generations $1-4$, and their linear polymer counterparts with 19,43 , 91, and 187 monomers per molecule under steady planar extensional flow, we compute the first extensional viscosity $\eta_{1}$, second extensional viscosity $\eta_{2}$, and extensional viscosity $\bar{\eta}$, which are expressed in terms of the components of the molecular pressure tensor $\mathbf{P}^{M}$ calculated as below

$$
\begin{gathered}
\eta_{1}=\left\langle\frac{P_{y y}-P_{x x}}{\dot{\epsilon}}\right\rangle, \\
\eta_{2}=\left\langle\frac{P_{y y}-P_{z z}}{\dot{\epsilon}}\right\rangle, \\
\bar{\eta}=\frac{\eta_{1}}{4} .
\end{gathered}
$$

The extensional viscosity is often nondimensionalized with respect to the shear viscosity. This ratio is called the Trouton ratio. In Eq. (11), the factor four comes from the Trouton ratio for planar flows. This implies that in the Newtonian regime, the planar extensional viscosity $\bar{\eta}$ is comparable to the shear viscosity and the first planar elongational viscosity $\eta_{1}$ is four times the shear viscosity.

Figure 2 shows the extensional viscosity $\bar{\eta}$ in the form of a logarithmic plot versus extension rate $\dot{\epsilon}$ for dendrimer, hyperbranched, and linear polymers with $19,43,91$, and 187 monomers per molecule. The overall trend includes three distinct regions: Initial plateau Newtonian behavior followed by a deformation thickening region, terminated by a considerable deformation thinning tail.

The first important issue to discuss here is the influence of the finite extensibility parameter of the FENE potential on the rheological behavior of our model polymer melt. Kisbaugh and McHugh (1990) studied the effect of this parameter on flow behavior of polymer solutions under shear flow. They showed that in the case of no finite extensibility (Hookean potential), viscosity will increase indefinitely as a function of strain rate, and there will be no thinning region after viscosity upturn occurs. This suggests finite extensibility leads to the second relaxation mechanism of the chain after being fully extended, namely, the overall alignment of the molecules at higher strain rates with respect to the flow fields.

In order to discuss the occurrence of these regions for different molecules, we subdivide our systems into (a) linear molecules including systems with entanglement (L187) [according to Kröger et al. (1993), entanglement molecular weight for linear molecules starts at approximately 100 monomers per chain] and linear molecules below the 

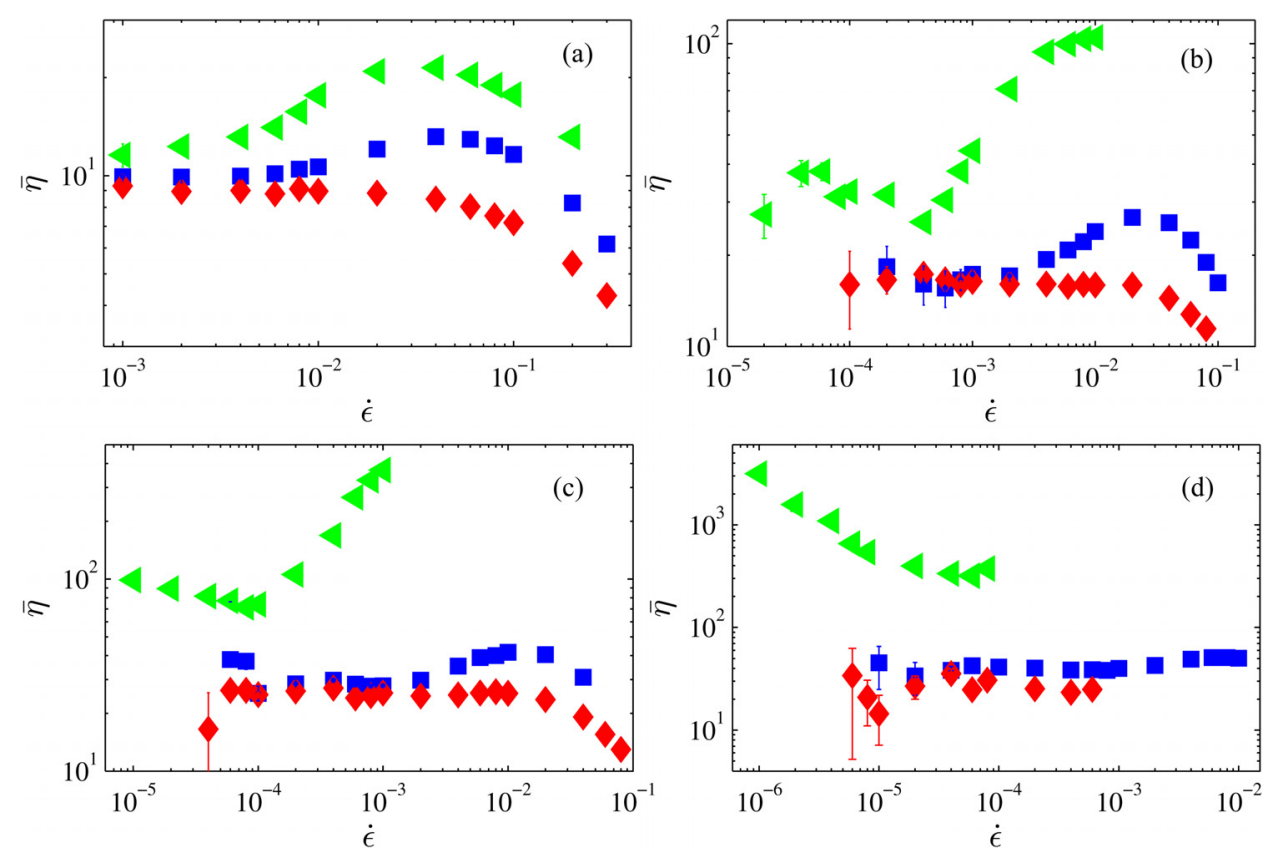

FIG. 2. Strain-rate dependence of extensional viscosity for linear (L), hyperbranched (H), and dendrimer (D) molecules with 19 (a), 43 (b), 91 (c), and 187 (d) monomers per molecule. Symbols in each subplot includes L $\rightarrow, \mathrm{H} \boldsymbol{\square}, \mathrm{D} \diamond$.

entanglement molecular weight (including L19, L43, and L91) and (b) dendrimers and hyperbranched molecules (D19, D43, D91, D187, H19, H43, H91, and H187).

For category (a) and generally for linear chains except L19, there is an extra thinning region at low strain rates before appearance of the thickening behavior [Figs. 2(b)-2(d)], and we suggest that this comes from the fact that linear chains can align themselves more easily along with the flow field than constrained dendritic structures even at fairly low strain rates. The intensity of this initial thinning increases with molecular weight, and as such, we do not see it for the L19 system, whereas there is a very strong initial thinning for L187 system. It should be mentioned that for the L187 system at the available strainrate window presented in Fig. 2(d), just the initial thinning region has been captured. Newtonian behavior for the L187 system happens at much lower strain rates and is not captured in this work due to the technical limitations which were mentioned earlier in Sec. II B. This trend is already reported in an experimental paper by Bhattacharjee et al. (2002) for entangled polymer solutions under uniaxial elongational flow. They suggested that for entangled polymers as $\dot{\epsilon}$ increases, the polymer chains become significantly aligned with the principle axis of strain, but they do not stretch, which causes the extensional viscosity to decrease. Once chain stretching begins, the viscosity upturn shows up. Therefore, thinning at lower values of $\dot{\epsilon}$ is characteristic of polymeric melts with a considerable number of entanglements per chain. For unentangled polymer melts or solutions, extensional viscosity increases monotonically from the limiting Trouton value of $4 \eta_{0}$ under PEF with increasing $\dot{\epsilon}$.

In the case of dendrimers and hyperbranched molecules, we might be able to interpret their elongational behavior based on their constrained topology which prevents them from being fully stretched and fully oriented along the flow direction. In addition, based on structural analysis later in this paper, we find that dendrimers and hyperbranched 
molecules change their size, shape, and orientation in the melt while undergoing elongational flow. We suggest that the stretched backbone/branches may be responsible for the thickening behavior at intermediate strain rates for linear/hyperbranched molecules, and global alignment is responsible for the thinning region for both dendrimers and hyperbranched molecules. In Fig. 2, one can also see that there is a significant difference between hyperbranched and dendrimer behavior under PEF, namely, the missing thickening region for dendrimers compared to hyperbranched molecules. This implies that imperfection in the microstructure of the hyperbranched molecules introduces some free volume throughout the interior of the molecules which facilitate the motion of branches in the hyperbranched structure compared to dendrimer architecture in which the tightly packed structure prevents branches being stretched very easily under PEF. Hence, due to the very globular structure and constrained geometry of dendrimers, their rheological behavior involves Newtonian behavior at low strain rates, with a weak viscosity upturn at moderate strain rates followed by a thinning region. The viscosity upturn decreases with the molecular weight and completely vanishes for the high molecular weight dendrimers for the strain rates studied here. The dampening of the viscosity upturn for dendrimers has been observed previously by Bosko and Prakash (2008) for shear flow. Neelov and Adolf studied the intrinsic viscosity of dendrimers (2003) and hyperbranched polymer (2004) solutions under elongational flow using Brownian dynamics simulations. They suggested that "as $\dot{\epsilon}$ increases, dendritic molecules first orient at low $\dot{\epsilon}$ as a whole along the flow axis without significant deformation and local orientation. Increasing $\dot{\epsilon}$ leads to local orientation at the level of the monomer leading to significant global deformation of dendrimer and hyperbranched molecules" [Neelov and Adolf (2003)].

Figure 3 shows the second extensional viscosity $\eta_{2}$ in the form of a logarithmic plot versus extension rate $\dot{\epsilon}$ for dendrimer, hyperbranched, and linear polymers with 19, 43,
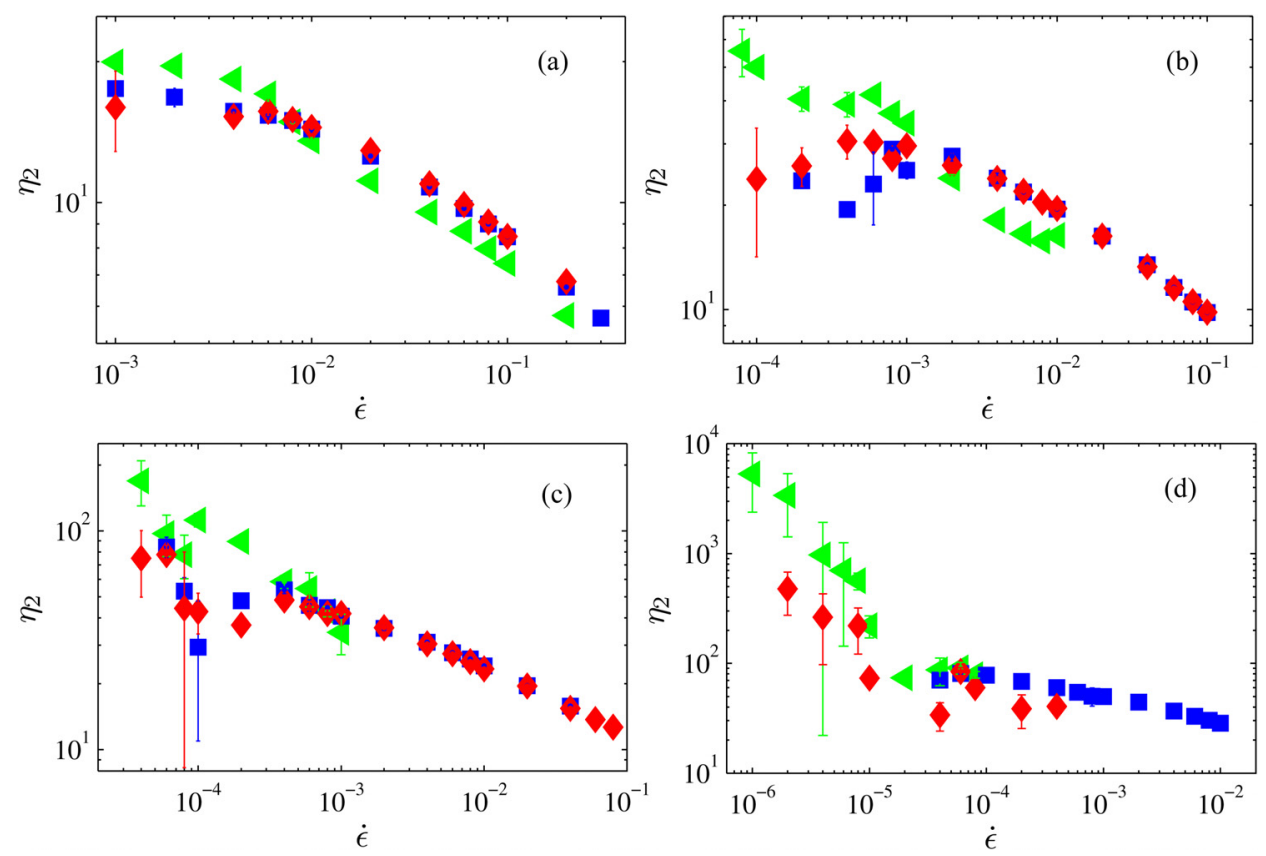

FIG. 3. Strain-rate dependence of second extensional viscosity for linear (L), hyperbranched $(\mathrm{H})$, and dendrimer (D) molecules with 19 (a), 43 (b), 91 (c), and 187 (d) monomers per molecule. Symbols in each subplot includes 
91, and 187 monomers per molecule. One can see that the start of the thinning region for all the systems is approximately around $\dot{\epsilon}=0.001$, which suggests that the elongation thinning behavior of $\eta_{2}$ is not sensitive to the molecular weight for these systems. The second planar elongational viscosity is very rarely measured, and the only available data are, e.g., Matin et al. (2003), Laun and Münstedt (1978), and Baig et al. (2006) for linear polymers. Our $\eta_{2}$ results are in qualitative agreement with the data of these papers.

Sarkar and Gupta (2001) used a modified version of the Carreau model [Bird et al. (1987)] for shear viscosity in order to predict the elongational viscosity as

$$
\eta_{1}=\eta_{0}\left[4+\delta\left\{1-\frac{1}{\sqrt{1+\left(\lambda_{1} I_{2}\right)^{2}}}\right\}\right]\left[1+\left(\lambda_{2} I_{2}\right)^{2}\right]^{\frac{m-1}{2}} .
$$

For $\delta=0$, the elongational viscosity in Eq. (12) reduces to the Carreau model with the elongational viscosity parameters $\lambda_{2}$ and $m$, respectively, replacing $\lambda$ and $n$ in the shear viscosity model. $I_{2}$ is the second scalar invariant of the strain rate tensor and is $8 \dot{\epsilon}^{2}$ for PEF and $2 \dot{\gamma}^{2}$ for shear flow. Physically, $I_{2}$ is a measure of the rate of viscous heat dissipation in steady state flow. The parameter $\lambda_{1}$ in Eq. (12) dictates $I_{2}$ for transition between Newtonian and elongation thickening regions of the viscosity against strain-rate curve, whereas $\delta$ characterizes the total increase in viscosity in the elongation thickening region. Parameters $\lambda_{2}$ and $m$ specify $I_{2}$ for the transition between elongation-thickening and power-law regions, and the power-law index for elongational viscosity, respectively. We used this model to compare our simulation results with and adjusted the parameters of the model using our simulation data, see Figs. 4(a)-4(d). The model parameters with our simulation data for dendrimers and hyperbranched polymers of length 19, 43, and 91 are provided in Table I.

Figure 4 reveals interesting aspects of elongational behavior of our studied systems. As opposed to shear flow, there is not just one time constant to define a single Weissenberg number. Here, we defined two Weissenberg numbers using two different time constants $\lambda_{2}$ and $\lambda_{1}$. Figures 4(a) and 4(b) show normalized extensional viscosity versus $\lambda_{2} I_{2}$ for dendrimer and hyperbranched systems, respectively. Figures 4(c) and 4(d) show normalized extensional viscosities against $\lambda_{1} I_{2}$ for dendrimers and hyperbranched systems, respectively. The former Weissenberg number $\lambda_{2} I_{2}$ makes the thinning tail of the plots fall on a single plot (partly only), while the latter $\lambda_{1} I_{2}$ makes the tickenning region of the plots collapse on a single plot (partly only). This is a very important aspect of the elongational flow, namely, that there is no single Weissenberg number to be used in order to plot a master curve. A combination of time constants will also merely show the plots in accord with the relative weightings of the constants and would be an arbitrary measure.

It is also important to note that, as discussed before, increasing perfection in the architecture of dendrimer molecules compared to hyperbranched polymers with imperfect structures, the thickening region tends to moderate and disappear for dendrimer systems by increasing the generation number. This is indicative of the constrained internal structure of these molecules that suppresses stretching of the branches along the flow lines. We suggest that this imperfection in hyperbranched polymers can produce some open spaces within the internal structure that can facilitate motion of the branches under flow so that molecules respond to the flow by stretching, which leads to increased intermolecular stress and hence the occurrence of viscosity upturn.

In order to find the viscous relaxation time under shear flow, it is common to use the following expression [Friedrich and Heymann (1988); Hartkamp et al. (2013)]: 

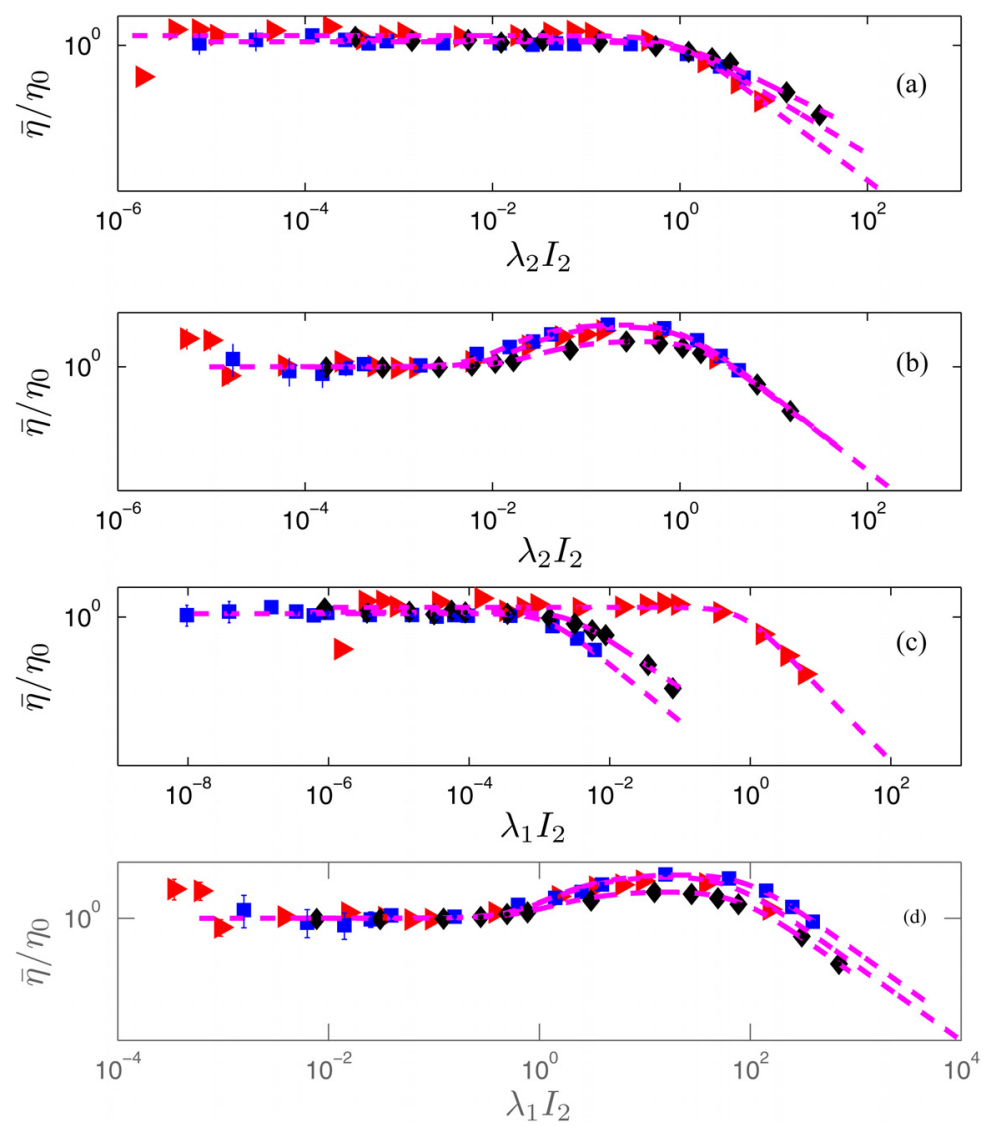

FIG. 4. Normalized extensional viscosity for dendrimers and hyperbranched polymers with 19,43 , and 91 monomers per molecule as a function of $\lambda_{2} I_{2}[(\mathrm{a})-(\mathrm{b})]$ and $\lambda_{1} I_{2}$ [(c)-(d)], respectively. Dashed lines (- -) show fitted data to the Sarkar-Gupta model. Symbols for each generation of dendrimers and hyperbranched molecules are as follows: 91 beads $\boldsymbol{\nabla}, 43$ beads $\mathbf{\square}, 19$ beads $\bullet$.

$$
\tau_{v}=\frac{\Psi_{1,0}}{2 \eta_{0}}
$$

where $\Psi_{1,0}$ is defined as the zero-shear rate first normal stress coefficient. We used data available in Le et al. (2009b) and Bosko et al. (2004b), for $\Psi_{1,0}$ and $\eta_{0}$, and calculated $\tau_{v}$ using Eq. (13). For more details, see Table II. These values are in good agreement with our elongation data calculated from the reciprocal of the strain-rate at which the thinning

TABLE I. Sarkar-Gupta model parameters.

\begin{tabular}{llrcl}
\hline \hline System & $\delta$ & \multicolumn{1}{c}{$\lambda_{1}$} & $\lambda_{2}$ & \multicolumn{1}{c}{$m$} \\
\hline DEN19 & 0.1 & 0.11 & 43.08 & 0.8 \\
DEN43 & 0.07 & 0.12 & 92.89 & 0.76 \\
DEN91 & 0.2 & 116.52 & 142.98 & 0.7 \\
HYP19 & 2.1 & 965.14 & 20.93 & 0.85 \\
HYP43 & 2.49 & 4905.12 & 52.94 & 0.782 \\
HYP91 & 2.38 & 11905.11 & 169.92 & 0.654 \\
\hline \hline
\end{tabular}


TABLE II. Relaxation times calculated from Eq. (13), $\tau_{v}$, reciprocal of $\dot{\epsilon}$ at which thinning region starts, $\tau_{p}$, and the Sarkar-Gupta model parameters, $\tau_{m}$.

\begin{tabular}{lccr}
\hline \hline System & $\tau_{v}$ & $\tau_{p}$ & $\tau_{m}$ \\
\hline DEN19 & - & 50 & 43.08 \\
DEN43 & - & 90 & 92.89 \\
DEN91 & - & 125 & 169.98 \\
HYP19 & $25(3)$ & 25 & 20.93 \\
HYP43 & $67(7)$ & 66.7 & 52.94 \\
HYP91 & - & 95 & 142.92 \\
LIN19 & - & 25 & 28.57 \\
LIN43 & - & 100 & - \\
\hline \hline
\end{tabular}

region begins $\left(\tau_{p}\right)$. Le et al. (2009b) and Bosko et al. (2004b) used the Carreau-Yasuda model to fit their shear-rate dependent shear viscosity data for hyperbranched polymers and dendrimers of generations 1-4, respectively, and calculated the adjustable parameters of the model. Values obtained for the time constant of this model only gave an approximation of the relaxation times and were of the same order of magnitude as the relaxation times calculated from the reciprocal of the strain-rate at which thinning begins. Likewise, values obtained for $\lambda_{2}$ from the Sarkar-Gupta model here $\left(\tau_{m}\right)$ are shown in Table II and are within the same order of magnitude of the viscous relaxation times calculated from Eq. (13) and also the reciprocal of the strain-rate at which thinning begins $\left(\tau_{p}\right)$ (for the extensional viscosity versus strain-rate plot). Unfortunately, the $\Psi_{1,0}$ and $\eta_{0}$ values are not available for all the systems under study due to poor signal to noise quality for these properties at very low strain-rates. In addition, the relaxation times obtained from the Sarkar-Gupta model parameters are shown in Fig. 5 as a function of molecular weight for dendrimers and hyperbranched molecules of generations $1-3$. Both dendrimers and

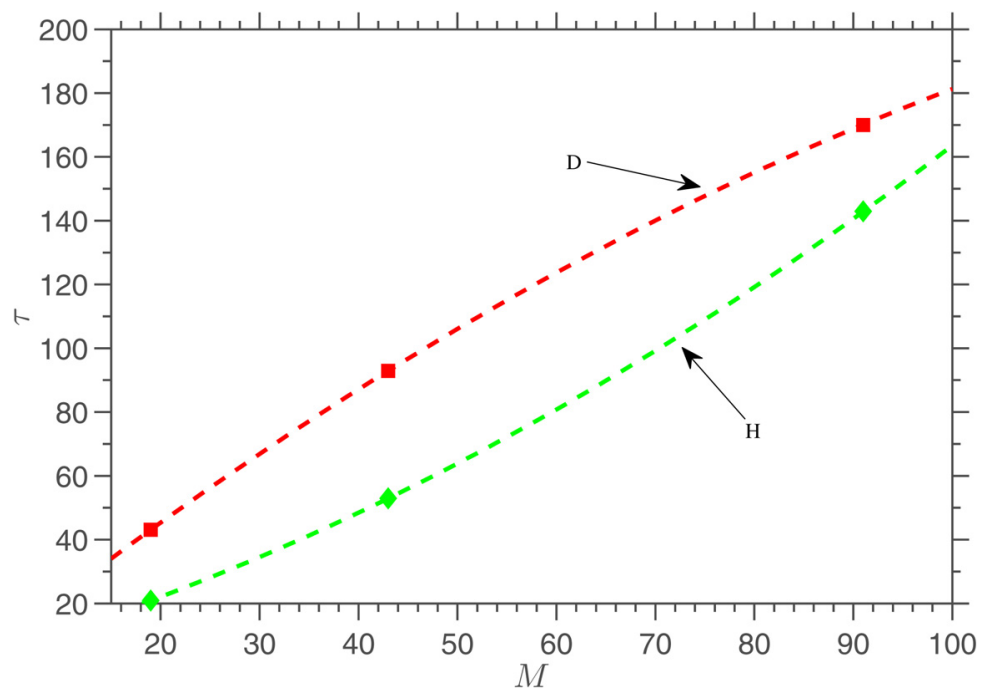

FIG. 5. The relaxation times obtained from the Sarkar-Gupta model parameters against molecular weight (in reduced unit) for dendrimers and hyperbranched molecules of generations 1-3. Symbols are as follows: dendrimers (D) $\mathbf{\square}$, hyperbranched $(\mathrm{H}) \bullet$. Dashed lines are quadradic fits to the data, which confirm the predicted relation $\tau \propto M^{2.2}$ [Rubinstein and Colby (2003)]. 
hyperbranched molecules show approximately quadratic dependence on the molecular weight under PEF, which confirms theoretical predictions $\tau \propto M^{2.2}$ for short branched polymers [Rubinstein and Colby (2003)]. This relation can be used to predict the approximate longest relaxation time for dendrimers and hyperbranched molecules with any molecular weight. Furthermore, the strain rate at which thinning behavior starts is obtainable from the reciprocal of this relaxation time. This trend is consistent with Bosko et al. (2006) for dendrimers under shear flow.

In order to compare differences in melt behavior of our systems under PEF with shear flow, we plot the normalized extensional viscosity $\bar{\eta} / \eta_{0}$ and corresponding shear viscosity results for hyperbranched molecules of generations $1-4$ along with dendrimer and linear counterparts under PEF as a function of $\lambda_{1} I_{2}$ in Fig. 6 . We find that for both PEF and shear flow under Newtonian flow, the expected normalized viscosity of 1 is obtained. Second, nonlinear behavior under both types of flow starts at the same $\lambda_{1} I_{2}$ value. This confirms the general knowledge that the Weissenberg number at the onset of extension thickening in PEF is roughly the same as the Weissenberg number at which the shear viscosity begins to shear thin under shear flow [Bird et al. (1987)].

\section{B. Structural analysis}

The extension of a molecule in space can be characterized by its radius of gyration. The average tensor of gyration is given by the expression

$$
\mathbf{R}_{g}^{2} \equiv\left\langle\frac{\Sigma_{\alpha=1}^{n} m_{\alpha}\left(\mathbf{r}_{\alpha}-\mathbf{r}_{C M}\right)\left(\mathbf{r}_{\alpha}-\mathbf{r}_{C M}\right)}{\Sigma_{\alpha=1}^{n} m_{\alpha}}\right\rangle,
$$

where $\mathbf{r}_{\alpha}$ is the position of monomer $\alpha, \mathbf{r}_{C M}$ is the position of the molecular center of mass, and the angle brackets denote an ensemble average. The value of the squared radius of gyration, which is defined as the trace of the tensor of gyration $\left(R_{g}^{2}=\operatorname{Tr}\left(\mathbf{R}_{g}^{2}\right)\right)$,

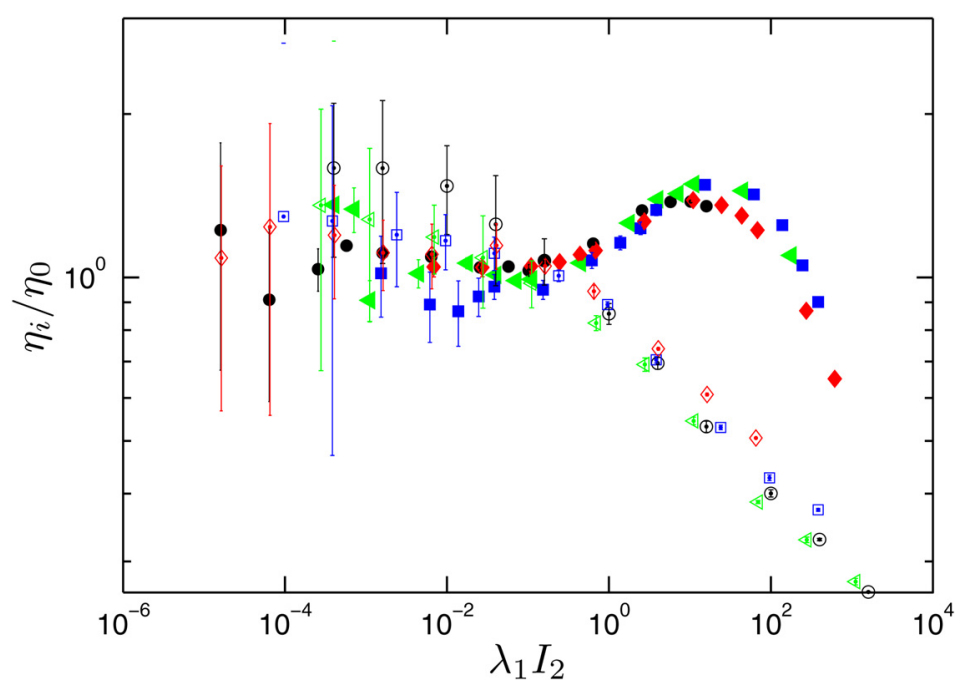

FIG. 6. Normalized planar elongational $\left(\eta_{i}=\bar{\eta}\right)$ and shear viscosities $(i=s) \eta_{s} / \eta_{0}$ for hyperbranched molecules of generations $1-4$ versus $\lambda_{1} I_{2}$. The coincidence of the zero shear rate viscosity and the zero extension rate viscosity in the Newtonian regime indicates the validity of our extensional flow simulations. Symbols under PEF are as follows: H187 $\bullet$, H91 $\downarrow$, H43 $\square$, H19 $\bullet$. Open symbols identify correspondent molecules under shear flow. 
characterizes the size of the molecule and can be compared with the experimentally measured radial sizes of dendrimers and hyperbranched molecules. Figure 7 illustrates the changes in shape and orientation of dendrimers with 91 monomers per molecule at equilibrium and over a range of strain rates under PEF. These snapshots suggest that at equilibrium, the molecule has its own natural globular configuration and under PEF, branches of the molecule stretch in the $x$-direction and compress in the $y$-direction. This results in the molecules becoming more flat and ellipsoidal in shape rather than spherical. More evidence for the tendency to become ellipsoidal in shape is given later in this section by calculating the eigenvalues of the tensor of gyration and also by schematic 3D representations of dendrimer, hyperbranched, and linear molecules under PEF and shear flow.

Figure 8 presents the dependence of the normalized radii of gyration with respect to its value at equilibrium $\left\langle R_{g}^{2}\right\rangle_{0}$ for dendrimer, hyperbranched, and linear polymers with $19,43,91$, and 187 monomers per molecule as a function of $I_{2}$ for elongational flow and also as a comparison for hyperbranched molecules undergoing shear flow. Under PEF, molecular size increases with $I_{2}$ for all the studied systems and tends to saturate at higher $I_{2}$ values. This means that even at low $I_{2}$ values, molecules are stretched under PEF which reveals the importance of the PEF flow field on molecular deformation. Under shear flow for all studied systems, at small values of $I_{2}$, the averaged size of the polymer molecules fluctuate around a constant value. It is only at intermediate to high $I_{2}$ regions that molecular size increases but is always smaller than its corresponding value under PEF. Differences between molecular size for hyperbranched molecules under PEF and shear flow become smaller as generation increases. This suggests that for higher generation of hyperbranched molecules, the type of flow becomes less influential on molecular size. By increasing the generation number, the interior of the molecule becomes increasingly crowded which makes stretching difficult.

Comparing different architectures reveals that linear molecules are always larger in size than dendritic molecules, and this difference in size increases as molecular weight

(a)

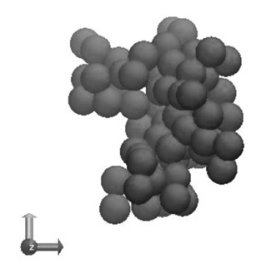

(b)

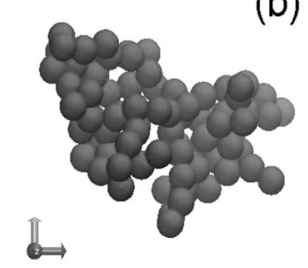

(d)

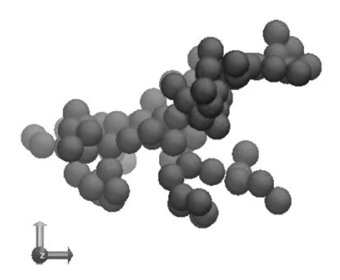

(c)

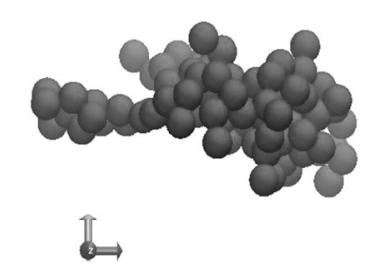

FIG. 7. Snapshot configuration of a dendrimer of generation 3 at (a) equilibrium, (b) $\dot{\epsilon}=0.0008$, (c) $\dot{\epsilon}=0.008$, and (d) $\dot{\epsilon}=0.08$. 

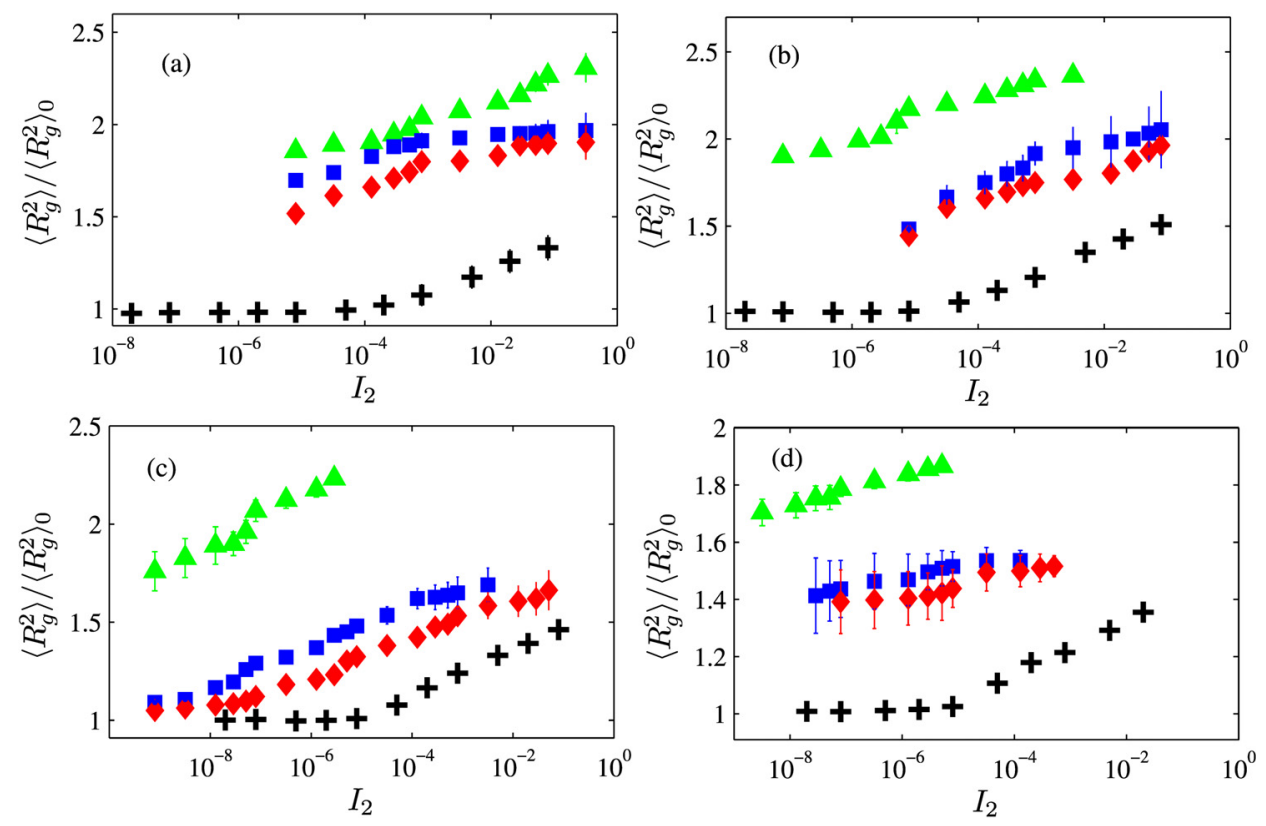

FIG. 8. Normalized mean squared radius of gyration of linear, hyperbranched, and dendrimer polymers with 19 (a), 43 (b), 91 (c), and 187 (d) monomers at different $I_{2}$, and also hyperbranched molecules under shear flow. Symbols under PEF are as follows: L $\boldsymbol{\Delta}, \mathrm{H} \boldsymbol{\boldsymbol { \square }}, \mathrm{D} \diamond$, and + for hyperbranched molecules under shear flow.

increases (see subplots of Fig. 8). The higher the generation number is, the more crowded the interior of the molecule becomes, which results in limited movement of the branches under both types of flow. The result is smaller increase in size for high molecular weight melts under any type of flow.

Hyperbranched molecules show higher radius of gyration compared to their corresponding dendrimer molecules. This was expected due to imperfection in hyperbranched molecule architecture compared to perfect globular architecture of dendrimers. Imperfection gives hyperbranched molecules this unique opportunity to use the available free volume in their interior to move and stretch more freely compared to constrained dendrimer microstructures, which results in their relatively larger size.

Neelov and Adolf studied structural properties of dendrimers (2003) and hyperbranched (2004) solutions under elongational flow using Brownian dynamics simulations. Their results are in qualitative agreement with data presented here for radius of gyration variations of both dendrimer and hyperbranched polymers. They found that the increase/upturn in radius of gyration is less pronounced for dendrimers compared to hyperbranched molecules and less pronounced for hyperbranched molecules compared to linear counterparts, which is comparable to our findings.

As the radius of gyration is an averaged quantity over the three directions, we can further investigate the deformation of molecules under both types of flow by calculating ratios of different eigenvalues of the tensor of gyration, which follows.

Further analysis of the tensor of gyration can provide insights into the shape and orientation of the molecules at different directions in space. For each system mentioned above, the eigenvalues of the tensor of gyration (in descending order, $L_{1}, L_{2}$, and $L_{3}$ ) were computed and averaged over the ensemble. These eigenvalues can be interpreted as the linear dimensions of the ellipsoid occupied by the average molecule, regardless of its orientation. Changes in these values with $I_{2}$ quantitatively describe the flow induced stretching 
of the molecules. In addition, the asymmetry of molecules is characterized by the ratio of the eigenvalues of the average gyration tensor, which we define here as $L_{12}=L_{1} / L_{2}$, $L_{13}=L_{1} / L_{3}$, and $L_{23}=L_{2} / L_{3}$. If the ratios of the eigenvalues are closer to 1 , the molecules have greater spherical symmetry. If $L_{1}>L_{2} \approx L_{3}$, then the shape is prolate and if $L_{1} \approx L_{2}>L_{3}$, the shape is considered as oblate. Figures 9-11 show the changes in these three ratios as a function of $I_{2}$. As one can see, all these three ratios are constant at small $I_{2}$ values and tend to increase at higher $I_{2}$. At very high $I_{2}$ values, they tend to decrease slightly. In addition, linear polymers compared to dendrimers and hyperbranched molecules show sharper increase, especially for $L_{13}$ ratios (Fig. 10), and this arises because dendrimers and hyperbranched polymers are structurally constrained and are not able to become fully stretched compared to linear chains. In addition, hyperbranched molecules show higher $L_{i j}$ values compared to dendrimers which implies they have a more flattened ellipsoidal shape than dendrimers.

We find that, at low to intermediate $I_{2}$ values, hyperbranched molecules show much lower $L_{i j}$ values under shear flow in comparison to PEF. Under shear flow and at lower $I_{2}$ values, these three ratios are closer to 1 , which means they have more spherical shape at low $I_{2}$. Under PEF, even at very low $I_{2}$ values, molecules have an elongated shape and stretched configuration, contrary to the spherical shape under shear flow. By further increasing $I_{2}$, under both types of flow, they become more elongated. Only at very high $I_{2}$ values do the eigenvalue ratios under shear flow dominate their corresponding values under PEF. It should be noted that at high values of $I_{2}$, molecules are at their fully stretched and aligned states under shear flow (see Fig. 6 for shear thinning region under shear flow). It is important to note that higher $L_{i j}$ ratios under shear flow at very high $I_{2}$ compared to PEF do not imply larger hyperbranched molecules under shear flow compared to PEF. Under shear flow, molecules start to deform from a spherical shape to an
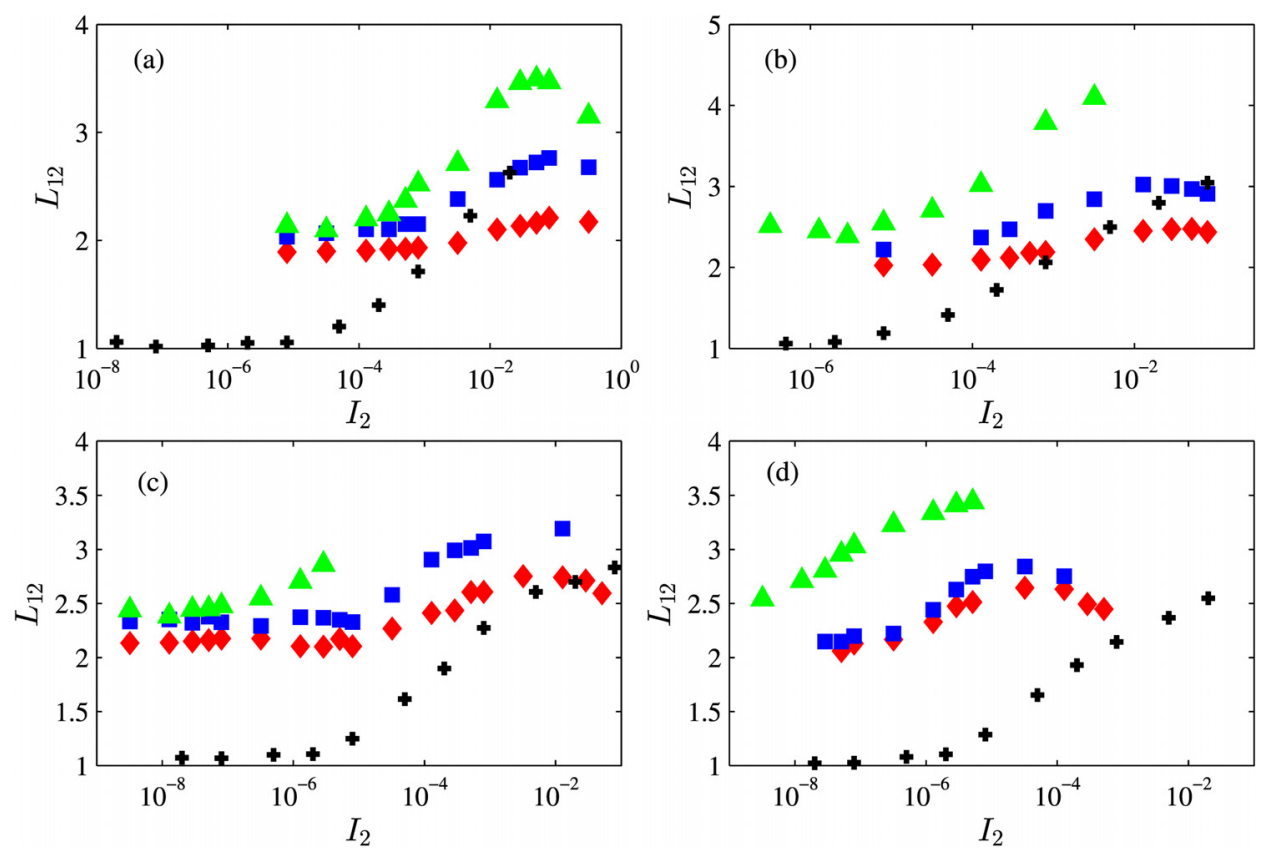

FIG. 9. $L_{12}$ ratios for linear, hyperbranched, and dendrimer polymers with 19 (a), 43 (b), 91 (c), and 187 (d) monomers per chain over a wide range of $I_{2}$ values. Also hyperbranched molecules under shear flow. Symbols under PEF are as follows: $\mathrm{L} \downarrow, \mathrm{H} \boldsymbol{\mathbf { Q }}, \mathrm{D} \bullet$, and + for hyperbranched molecules under shear flow. 

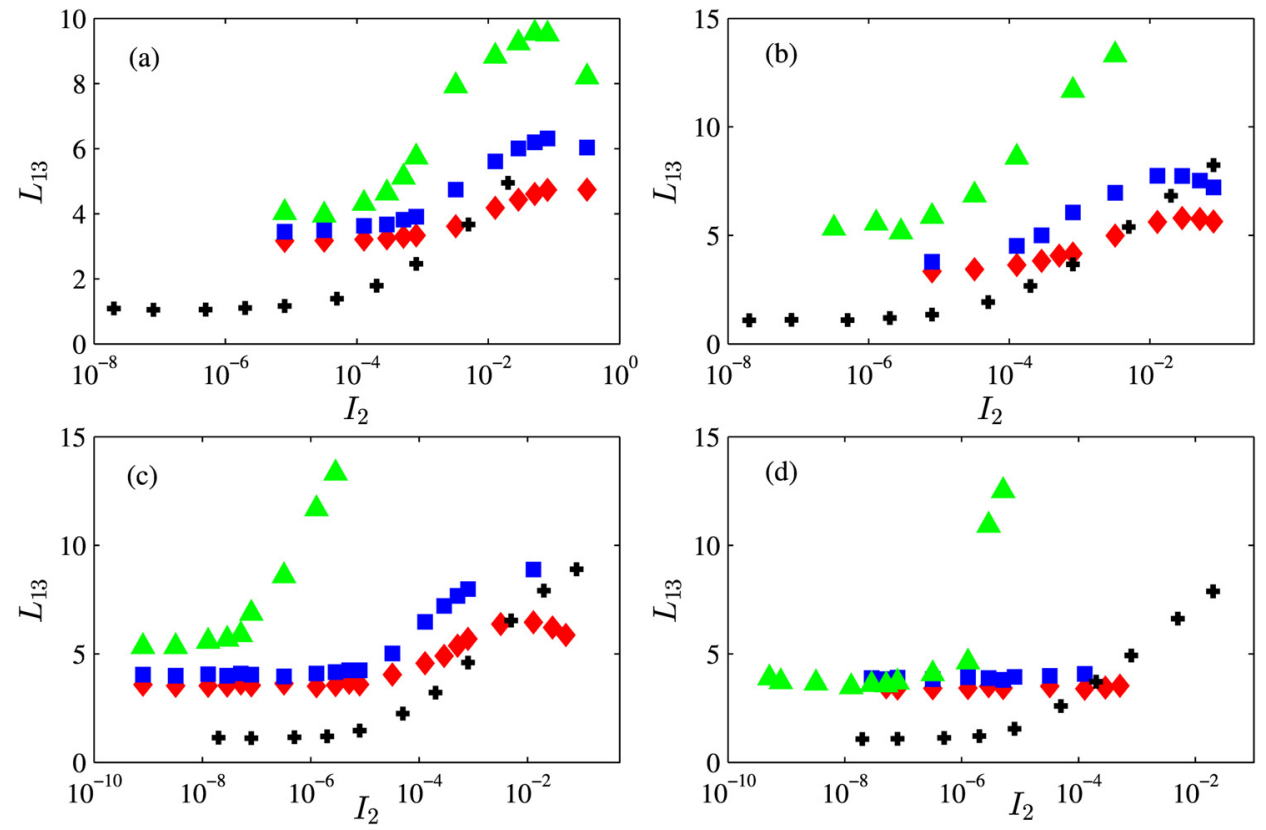

FIG. 10. $L_{13}$ ratios for linear, hyperbranched, and dendrimer polymers with 19 (a), 43 (b), 91 (c), and 187 (d) monomers per chain over a wide range of $I_{2}$ values. Also hyperbranched molecules under shear flow. Symbols under PEF are as follows: L $\downarrow$, H $\mathbf{\square}, \mathrm{D} \bullet$, and + for hyperbranched molecules under shear flow.
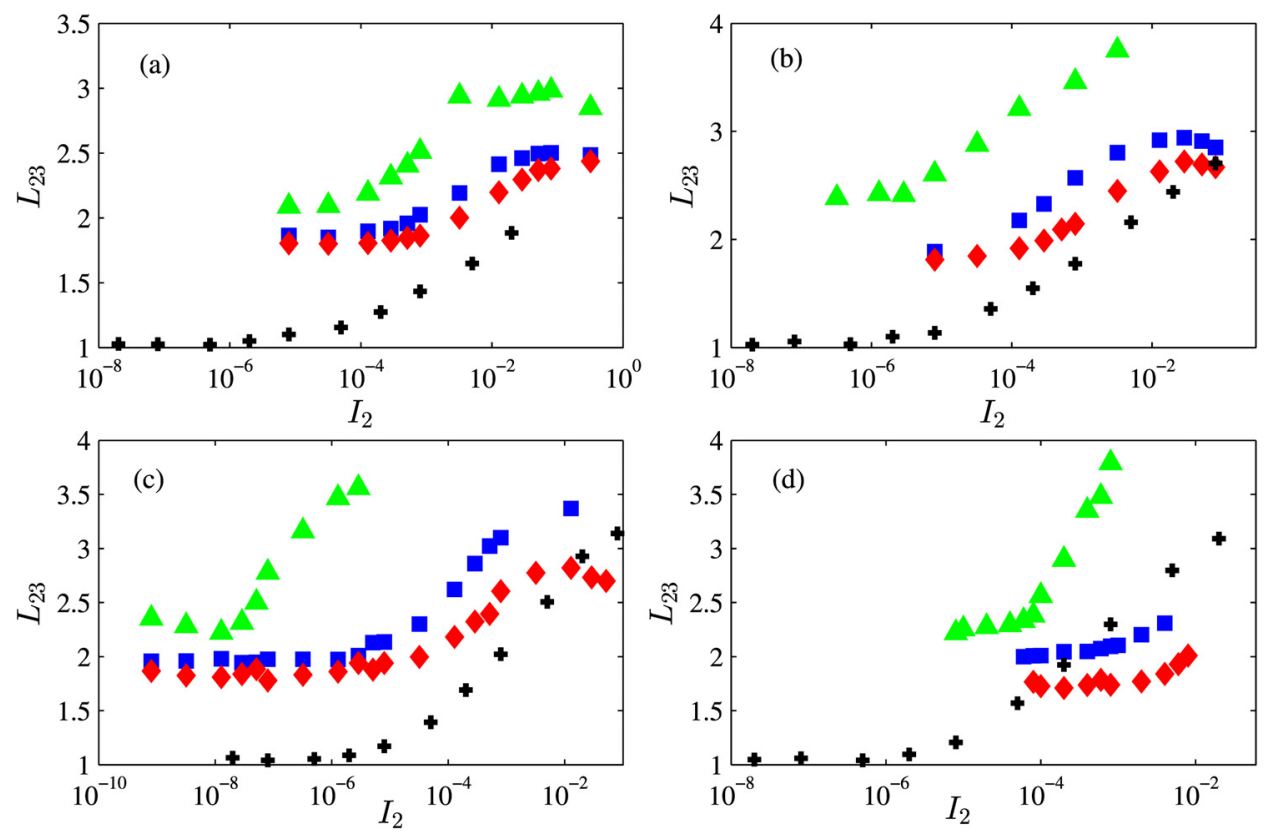

FIG. 11. $L_{23}$ ratios for linear, hyperbranched, and dendrimer polymers with 19 (a), 43 (b), 91 (c), and 187 (d) monomers per chain over a wide range of $I_{2}$ values. Also hyperbranched molecules under shear flow. Symbols under PEF are as follows: L $\downarrow, \mathrm{H} \mathbf{\square}, \mathrm{D} \bullet$, and + for hyperbranched molecules under shear flow. 
ellipsoidal shape at these high values of $I_{2}$, which leads to sharp increase in $L_{i j}$ values. In contrast to shear flow, under PEF molecules are already ellipsoidal, even at lower $I_{2}$ values, which leads to a gradual increase in $L_{i j}$ values and lower $L_{i j}$ under PEF compared to shear flow at very high $I_{2}$.

We find that there is a specific strain rate at which these ratios start to increase. The value of this strain rate decreases as the molecular weight increases. These values are listed in Table III and will be discussed in greater detail in what follows. Also, we find that the magnitude of increase in $L_{13}$ is much higher than $L_{12}$ and $L_{23}$. It means that the difference between the smallest $\left(L_{3}\right)$ and largest $\left(L_{1}\right)$ dimensions of the molecules becomes larger as strain rates increases. This is evidence that as strain rates increases, the molecules become much more elongated and flattened in shape.

To correlate flow-induced molecular deformation with viscoelastic behavior of the studied systems is of particular importance. Comparing $I_{2}$ at which the eigenvalue ratios start to rise (see Table III) along with $I_{2}$ at which viscosity upturn occurs provides us with very important information. We find that viscosity upturn occurs approximately at the same $I_{2}$ value where the eigenvalues start to rise. This indicates that the onset of molecular deformation under PEF coincides with the crossover from the Newtonian plateau to the non-Newtonian region in the viscosity curves. In addition, $\left\langle R_{g}^{2}\right\rangle$ saturates at $I_{2}$ values comparable to $I_{2}$ at which the ratios of different eigenvalues reach their maximum plateau. At this state, as has already been discussed, the molecules have a flattened ellipsoidal configuration. Also, thinning occurs at higher $I_{2}$ values when deformed molecules align themselves with the flow direction. Bosko et al. (2004a, 2004b) studied dendrimers under shear flow and found the same behavior. They concluded that flow-induced deformation is the major mechanism for the observed viscoelastic behavior of these molecules under shear, and molecular alignment, which occurs at all $I_{2}$ values, is a less effective mechanism at low to intermediate $I_{2}$ values.

In Fig. 12, we visualize the flow-induced deformation of dendrimers and linear molecules under PEF, and hyperbranched molecules under both shear and PEF in terms of $L_{i}$ values as linear dimensions of the ellipsoid occupied by the average molecule. By comparing different eigenvalue ratios, we find that linear molecules at low $I_{2}$ have a prolate shape which changes to a much more flattened ellipsoidal shape as $I_{2}$ increases. Hyperbranched molecules under shear flow have a spherical shape at low $I_{2}$ and at high

TABLE III. Strain-rates at which thinning $\dot{\epsilon}_{t h}$ and upturn start to occur for elongational viscosity $\dot{\epsilon}_{u t}$, ratios of different eigenvalues $\dot{\epsilon}_{L_{12 i}}, \dot{\epsilon}_{L_{13 i}}, \dot{\epsilon}_{L_{23 i}}$, and also strain-rate at which thinning occurs in which they show maximum or a plateau behavior $\dot{\epsilon}_{L_{12 m}}, \dot{\epsilon}_{L_{13 m}}$, and $\dot{\epsilon}_{L_{23 m}}$.

\begin{tabular}{lcccccccc}
\hline \hline System & $\dot{\epsilon}_{t h}$ & $\dot{\epsilon}_{u t}$ & $\dot{\epsilon}_{L_{12 i}}$ & $\dot{\epsilon}_{L_{13 i}}$ & $\dot{\epsilon}_{L_{23 i}}$ & $\dot{\epsilon}_{L_{12 m}}$ & $\dot{\epsilon}_{L_{13 m}}$ & $\dot{\epsilon}_{L_{23 m}}$ \\
\hline DEN19 & 0.02 & - & 0.02 & 0.01 & 0.01 & 0.1 & 0.1 & 0.04 \\
DEN43 & 0.01 & - & 0.02 & 0.002 & 0.004 & 0.04 & 0.04 & 0.06 \\
DEN91 & 0.008 & - & 0.002 & 0.002 & 0.002 & 0.02 & 0.04 & 0.04 \\
DEN187 & - & - & 0.0004 & - & 0.004 & 0.002 & - & - \\
HYP19 & 0.04 & 0.006 & 0.015 & 0.008 & 0.008 & 0.1 & 0.1 & 0.04 \\
HYP43 & 0.015 & 0.004 & 0.006 & 0.002 & 0.002 & 0.04 & 0.04 & 0.06 \\
HYP91 & 0.011 & 0.002 & 0.002 & 0.002 & 0.002 & 0.02 & 0.04 & 0.04 \\
HYP187 & - & 0.001 & 0.0004 & - & 0.002 & 0.002 & - & - \\
LIN19 & 0.04 & 0.002 & 0.004 & 0.006 & 0.004 & 0.08 & 0.08 & 0.04 \\
LIN43 & 0.01 & 0.0006 & 0.01 & 0.001 & 0.001 & 0.02 & 0.02 & - \\
LIN91 & - & - & 0.00006 & 0.00008 & 0.00006 & - & - & - \\
LIN187 & - & - & 0.00004 & 0.0001 & 0.0001 & 0.0008 & - & - \\
\hline \hline
\end{tabular}


(a) D43, PEF

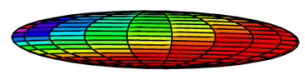

(c) $\mathrm{H} 43, \mathrm{PEF}$

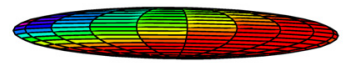

(e) H43, Shear

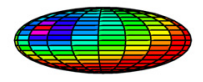

(g) L43, PEF (b) $\mathrm{D} 43, \mathrm{PEF}$

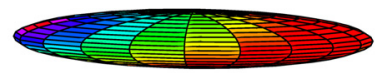

(d) $\mathrm{H} 43, \mathrm{PEF}$

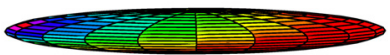

(f) H43, Shear

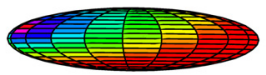

(h) L43, PEF

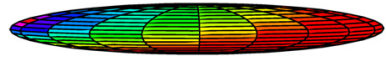

FIG. 12. Schematic ellipsoids for dendrimer, hyperbranched, and linear chains with 91 monomers under PEF at low (left column) $I_{2}=8 \times 10^{-8}$ and high (right column) $I_{2}=0.0512$. For comparison, we also show the hyperbranched system under shear flow at the same $I_{2}$ values. Colors are for clarity of perspective viewing and have no physical meaning.

$I_{2}$ deform to a prolate shape. Hyperbranched molecules under PEF, even at low $I_{2}$, have an ellipsoidal shape which becomes more flattened and prolate at higher $I_{2}$ values. While dendrimers have the same trend under PEF, they are less flattened compared to hyperbranched molecules under the same flow condition.

To analyze the distribution of mass within the molecule, the distribution of the monomers from the core (central monomer) has been used and is defined by

$$
g_{\text {core }}(\mathbf{r})=\left\langle\frac{\Sigma_{i=1}^{N} \Sigma_{\alpha=2}^{N_{\alpha}} \delta\left(\left|\mathbf{r}-\left(\mathbf{r}_{i \alpha}-\mathbf{r}_{i 1}\right)\right|\right)}{N}\right\rangle,
$$

where $\mathbf{r}_{i 1}$ is the position of the core and $\alpha$ runs over all the monomers belonging to the same molecule. The distribution of mass from the central unit (core) for hyperbranched and dendrimer molecules with 19, 43, and 91 monomers per molecule at the high strain-rate of $\dot{\epsilon}=0.004$ and also for dendrimer and hyperbranched molecules of 43 monomers at three different strain rates corresponding to the three different regions of rheological behavior can be found in Figs. 13 and 14, respectively. The first thing to note is the correlation between the position of the core and its neighbor, which is evident through a strong peak at the distance equal to the averaged bond length for all the generations. Second, by increasing the generation number (i.e., layers of branches of the microstructure of dendrimers and hyperbranched molecules), we see multiple peaks in the distribution from the core, which is more significant in the case of hyperbranched molecules and is indicative of treelike growth of layers in these molecules. For example, regardless of the first peak, we see one more peak for hyperbranched and dendrimer molecules with 19 beads (representing generation 1) and so on. These peaks are representative of areas that are accumulated by different layers of monomers within the interior of the molecular architecture of dendritic molecules. In Fig. 13, one can see that by increasing the strain rate, as the molecules are stretched in the flow, the distribution of mass becomes broader. We also observe that the average distance of the monomers from the 

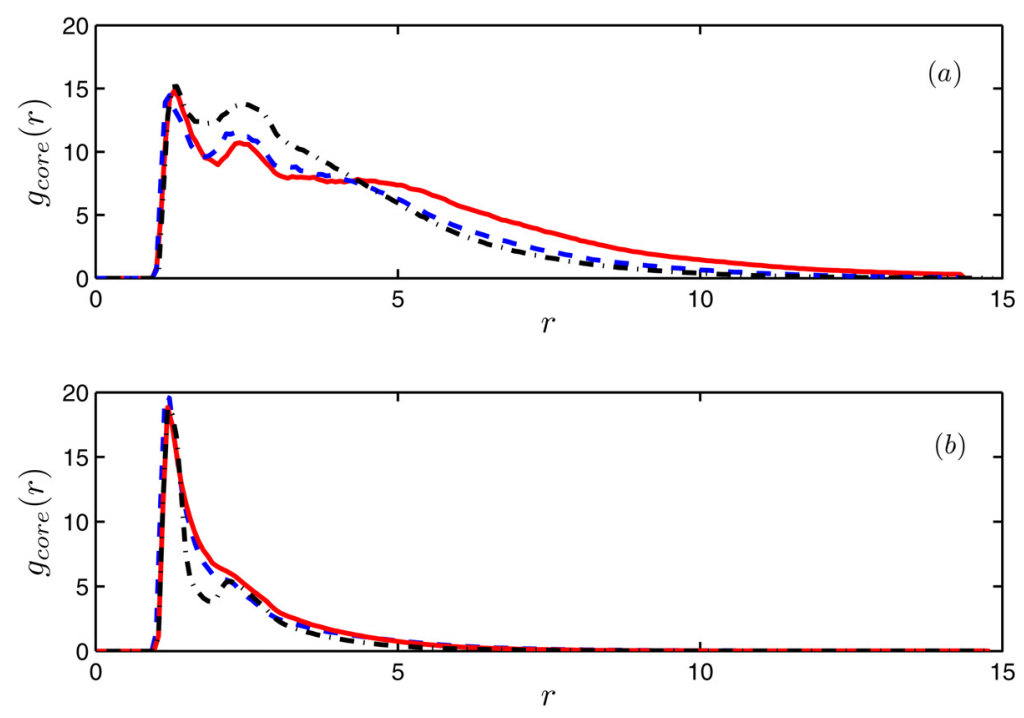

FIG. 13. Distribution of monomers from the core for hyperbranched (a) and dendrimer (b) with 43 monomers at three different strain rates. Symbols used for different strain rates are as follows: $\dot{\epsilon}=0.08-, \dot{\epsilon}=0.01--$, and $\dot{\epsilon}=0.004 \cdot-$.

core increases with strain rate. For example, at the highest strain rate, the distances of the monomers from the core for D43 and D91 are 7.2 and 13.6. However, these are smaller than the fully stretched arm, which are about 10.67 and 14.87 for D43 and D91, respectively. The difference between dendrimer and hyperbranched molecules is evident through the distribution at $\dot{\epsilon}=0.004$, where dendrimers show very narrow distribution, while for hyperbranched molecules, the distribution is broader. This stems from the fact that hyperbranched molecules can become stretched more easily than dendrimers due to their more open structure. Furthermore, for hyperbranched molecules one can see that this strain-rate $(\dot{\epsilon}=0.004)$ coincides with the maximum in the viscosity value, while for
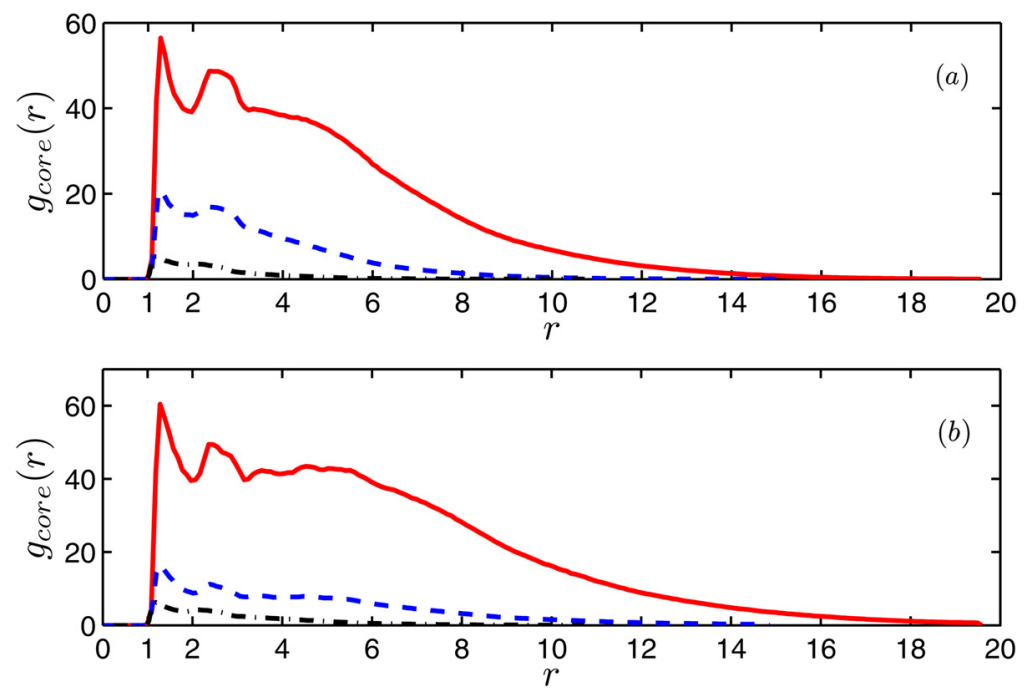

FIG. 14. Distribution of monomers from the core for hyperbranched (a) and dendrimers (b) with 19, 43, and 91 monomers at $\dot{\epsilon}=0.004$. Symbols used for different generations are as follows: 91 beads —, 43 beads - -, and 19 beads - . 
dendrimers, there is no maximum for the viscosity at this strain rate. These results are in full agreement with previous results reported by Le et al. (2009a, 2009b) and Bosko et al . (2004a, 2004b). Figure 14 displays the distribution from the core of dendrimers and hyperbranched polymers as a function of molecular weight and strain rate for comparative purposes.

One of the characteristic features of dendrimers and hyperbranched polymers is the large number of their terminal groups. Their number doubles with every generation, and their chemical activity can be controlled in the process of synthesis of the molecule. From the configurational point of view, it is important to understand the spatial distribution of these groups because the location of these groups over the molecule affects its chemical activity and physical properties. Similar to the bead distribution function presented earlier, the distribution of terminal groups is defined by

$$
g_{\text {term }}(\mathbf{r})=\left\langle\frac{\Sigma_{i=1}^{N} \Sigma_{\alpha} \delta\left(\left|\mathbf{r}-\left(\mathbf{r}_{i \alpha}-\mathbf{r}_{i 1}\right)\right|\right)}{4 \pi r^{2} N}\right\rangle,
$$

where in this case $\alpha$ runs over outermost monomers only.

In Fig. 15, we present the distribution of terminal groups from the central unit for dendrimers and hyperbranched polymers with 19, 43, and 91 monomers per molecule at strain rate of $\dot{\epsilon}=0.01$. For all the systems, secondary maxima are observed in the plot which indicates that not all of the end groups are located on the surface of the molecules, but rather that they can be found across the full range of distances from the central unit due to backfolding. This means that terminal groups exist everywhere throughout the interior of the molecules. This is consistent with other experimental results by Bodnar et al. (2000) and simulation results by Timoshenko et al. (2002), Le et al. (2009a), and Bosko et al. (2004a) under shear flow that have shown similar backfolding behavior with peaks observed in the plot of end groups. In comparison to the shear results of these authors, we conclude that backfolding phenomenon occurs under both shear and PEF flows, but it is more intense under PEF compared to shear flow.
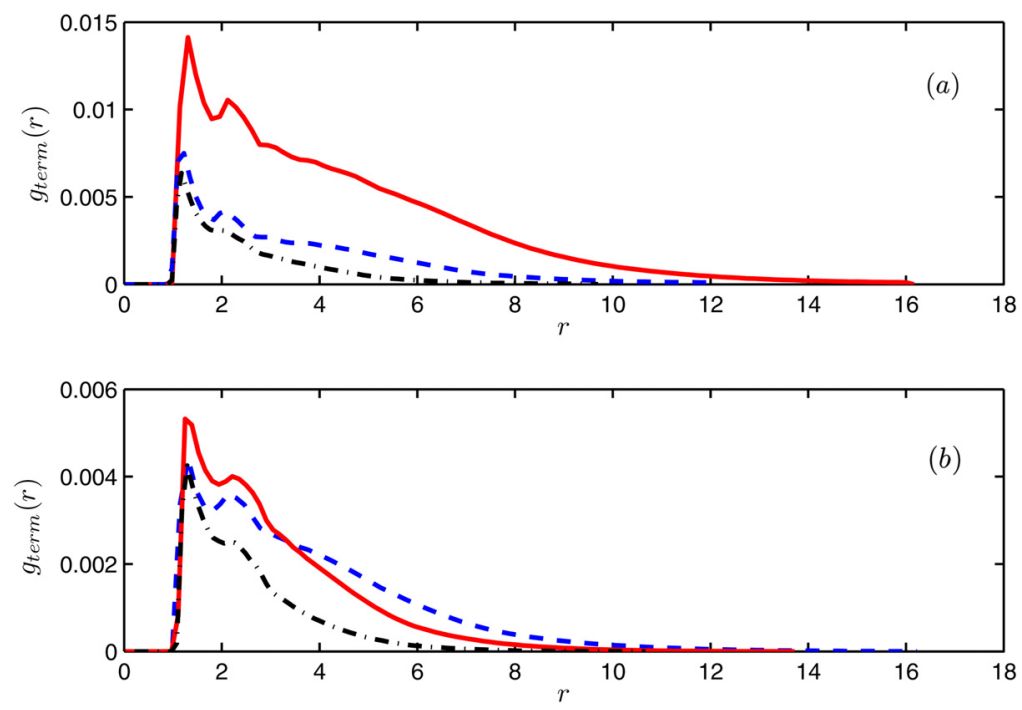

FIG. 15. Distribution of terminal groups for hyperbranched (a) and dendrimers (b) with 19, 43, and 91 monomers at high strain rate of $\dot{\epsilon}=0.01$. Symbols used for different generations are as follows: 91 beads —, 43 beads - -, and 19 beads $\cdot-$. 


\section{CONCLUSION}

The planar elongational melt rheology and structural properties of dendrimers and hyperbranched polymer melts of different molecular weights (generations 1-4) and their linear counterparts have been studied using a coarse-grained model and NEMD simulation techniques coupled with the so-called KR periodic boundary conditions in the NPT ensemble. According to the above rheological results, it is clear that dendrimers and hyperbranched polymers, due to their unique and compact structures, show much lower melt viscosities in comparison to their linear counterparts, which make them potentially useful candidates as flow modifiers and flow aid agents. The rheological properties, including extensional viscosity and second extensional viscosity, were calculated. Similar to previous experimental and simulation data for linear polymer melts, the elongational viscosity showed three distinctive regions of behavior for dendrimers and hyperbranched melts: An initial Newtonian region, followed by a thickening region, and terminated by a thinning region at high strain-rates. Dendrimers, contrary to hyperbranched molecules, did not show a thickening region in their rheological behavior at the rates of strain studied. We related this phenomenon to the structural perfection of dendrimers compared with hyperbranched molecules, which caused reduced mobility of the branches and also reduced flow-induced stretching and alignment of the molecules. Imperfection in the architecture of hyperbranched molecules introduced some free volumes that facilitated the movement and stretching of the branches. We suggest that this stretching is responsible for the thickening behavior of hyperbranched molecules. Furthermore, terminal thinning behavior is related to the finite extensibility of the FENE potential and flow-induced alignment. In addition, we fitted our simulation results with the Sarkar-Gupta model to calculate the adjustable parameters of this model from our normalized extensional viscosity data over a wide range of strain-rates. The longest relaxation times for dendrimers and hyperbranched molecules were calculated from the model parameters and were in a good agreement with those calculated from the zero-shear rate normal stress coefficients.

In addition to the rheological behavior, structural changes under PEF and shear flow were analyzed using different standard distribution functions and also eigenvalues of the tensor of gyration. The ratios of the eigenvalues demonstrated that, under shear flow, hyperbranched molecules change their shape from a spherical conformation to a prolate conformation as strain rate increases. Under PEF, even at low strain rates, molecules were ellipsoidal in shape and by increasing the elongation rate, they became much more flattened and prolate. Dendrimers showed the same changes in their shape, but they were less flattened. Linear molecules were more elongated compared to hyperbranched and dendrimer molecules at low elongation rates, while changing to a much more flattened ellipsoidal shape at higher strain rates. We also studied the distribution of monomers from the core of the molecules under PEF at three different strain rates corresponding to the three different regions of the rheological behavior of dendrimers and hyperbranched molecules. Generally, the distance of monomers from the core increases more significantly for hyperbranched molecules compared to dendrimers. For dendrimers, at small strain rates (i.e., the initial Newtonian region), the distribution of monomers from the core was very narrow and at intermediate strain rates became slightly broader. Only at very high strain rates did the distance of monomers from the core increase significantly. This broadening of the distribution corresponds to the thinning region of the viscosity curve. For hyperbranched molecules, even at low strain rates, the distribution of monomers is very broad. This arises from the fact that hyperbranched molecules can stretch and align themselves in the flow direction more easily than dendrimers due to their less 
constrained molecular architecture. Finally, we showed the existence of the typical backfolding phenomena for dendrimers and hyperbranched polymers under PEF. End groups were found across the full range of distances within the interior of the molecules rather than just at the outermost regions of the molecules.

\section{ACKNOWLEDGMENTS}

The authors thank Victorian Partnership for Advanced Computing for a grant of supercomputer time. They would also like to thank the referees for valuable suggestions which have improved the paper.

\section{References}

Aust, C., M. Kröger, and S. Hess, "Structure and dynamics of dilute polymer solutions under shear flow via nonequilibrium molecular dynamics," Macromolecules 32, 5660-5672 (1999).

Baig, C., B. J. Edwards, D. J. Keffer, and H. D. Cochran, "Rheological and structural studies of linear polyethylene melts under planar elongational flow using nonequilibrium molecular dynamics simulations," J. Chem. Phys. 124, 084902 (2006).

Baranyai, A., and P. Cummings, "Steady state simulations of planar elongational flow by nonequilibrium molecular dynamics," J. Chem. Phys. 110, 42-45 (1999).

Bhattacharjee, P. K., J. P. Oberhauser, G. H. McKinley, L. G. Leal, and T. Sridhar, "Extensional rheometry of entangled solutions,” Macromolecules 35, 10131-10148 (2002).

Bird, R. B., R. C. Armstrong, and O. Hassager, Dynamics of Polymeric Liquids, Volume I, Fluid Mechanics (Wiley, New York, 1987).

Bodnar, I., A. S. Silva, R. W. Deitcher, N. E. Weisman, Y. H. Kim, and N. J. Wagner, "Structure and rheology of hyperbranched and dendritic polymers. I. Modification and characterization of poly(propyleneimine) dendrimers with acetyl groups,” J. Polym. Sci., Part B: Polym. Phys. 38, 857-873 (2000).

Bosko, J., B. D. Todd, and R. J. Sadus, "Internal structure of dendrimers in the melt under shear: A molecular dynamics study," J. Chem. Phys. 121, 1091-1096 (2004a).

Bosko, J. T., B. D. Todd, and R. J. Sadus, "Viscoelastic properties of dendrimers in the melt from nonequlibrium molecular dynamics,” J. Chem. Phys. 121, 12050-12059 (2004b).

Bosko, J., B. D. Todd, and R. J. Sadus, “Analysis of the shape of dendrimers under shear,” J. Chem. Phys. 124, 044910 (2006).

Bosko, J. T., and J. R. Prakash, "Effect of molecular topology on the transport properties of dendrimers in dilute solution at $\Theta$ temperature: A Brownian dynamics study,” J Chem. Phys. 128, 034902 (2008).

Daivis, P. J., and Evans, D. J., "Comparison of constant-pressure and constant volume nonequilibrium simulations of sheared model decane,” J. Chem. Phys. 100, 541-547 (1994).

Daivis, P. J., J. Evans, and J. P. Morriss, "Computer simulation study of the comparative rheology of branched and linear alkanes,” J. Chem. Phys. 97, 616-627 (1992).

Daivis, P. J., M. L. Matin, and B. D. Todd, "Nonlinear shear and elongational rheology of polymer melts at low srain rates," J. Non-Newtonian Fluid Mech. 147, 35-44 (2007).

Dealy, J. M., "Extensional flow of non-Newtonian fluids,” Polym. Eng. Sci. 11, 433-445 (1971).

Frascoli, F., and B. D. Todd, "Molecular dynamics simulation of planar elongational flow at constant pressure and constant temperature," J. Chem. Phys. 126, 044506 (2007).

Frechet, J. M. J., M. Hemmi, I. Gitsov, S. Aoshima, M. R. Leduc, and R. B. Grubbs, "Functional polymers and dendrimers: Reactivity, molecular architecture, and interfacial energy," Science 263, 1710-1715 (1994).

Frick, B., and D. Richter, "The microscopic basis of the glass transition in polymers from neutron scattering studies," Science 267, 1939-1945 (1995).

Friedrich, C., and L. Heymann, "Primary normal-stress coefficient prediction at high shear rates," Rheol. Acta 27, 567-574 (1988). 
Flory, P. J., "Molecular size distribution in three dimensional polymers. I. Gelation,” J. Am. Chem. Soc. 63, 3083-3090 (1941).

Gaborieau, M., and P. Castignolles, "Size-exclusion chromatography (SEC) of branched polymers and polysaccharides,” Anal. Bioanal. Chem. 399, 1413-1423 (2011).

Hartkamp, R., P. J. Daivis, and B. D. Todd, "Density dependence of the stress relaxation function of a simple fluid,” Phys. Rev. E 87, 032155 (2013).

Cifre, J. G. H., and J. G. de la Torre, "Steady-state behavior of dilute polymers in elongational flow. Dependence of the critical elongational rate on chain length, hydrodynamic interaction, and excluded volume," J. Rheol. 43, 339-358 (1999).

Hess, B., "Determining the shear viscosity of model liquids from molecular dynamics simulations," J. Chem. Phys. 116, 209-217 (2002).

Heyes, D. M., "Molecular dynamics simulations of extensional, sheet and unidirectional flow," Chem. Phys. 98, 15-27 (1985).

Hoover, W. M. G., and C. G. Hoover, "Links between microscopic and macroscopic fluid mechanics," Mol. Phys. 101, 1559-1573 (2003).

Hounkonnou, M. N., C. Pierleoni, and J. P. Ryckaert, "Liquid chlorine in shear and elongational flows: A nonequilibrium molecular dynamics study,” J. Chem. Phys. 97, 9335-9344 (1992).

Hsieh, T.-T., C. Tiu, and G. P. Simon, "Melt rheology of aliphatic hyperbranched polyesters with various molecular weights," Polymer 42, 1931-1939 (2001).

Ilg, P., and M. Kröger, "Molecularly derived constitutive equation for low-molecular polymer melts from thermodynamically guided simulation,” J. Rheol. 55, 69-93 (2011).

Kalra, V., and Y. L. Joo, "Coarse-grained molecular dynamics study of block copolymer/nanoparticle composites under elongational flow," J. Chem. Phys. 131, 214904 (2009).

Karayiannis, N. Ch., and V. G. Mavrantzas, "Hierarchical modelling of the dynamics of polymers with a nonlinear molecular architecture: Calculation of branch point friction and chain reptation time of H-shaped polyethylene melts from long molecular dynamics simulations," Macromolecules 38, 8583-8596 (2005).

Kim, Y. H., and O. W. Webster, "Water soluble hyperbranched polyphenylene: A unimolecular micelle?," J. Am. Chem. Soc. 112, 4592-4593 (1990).

Kisbaugh, A. J., and A. J. McHugh, "A discussion of shear-thickening in bead-spring models," J. NonNewtonian Fluid Mech. 34, 181-206 (1990).

Kraynik, A. M., and D. A. Reinelt, "Extensional motions of spatially periodic lattices," Int. J. Multiphase Flow 18, 1045-1059 (1992).

Kremer, K., and G. S. Grest, "Dynamics of entangled linear polymer melts: A molecular-dynamics simulation," J. Chem. Phys. 92, 5057-5086 (1990).

Kröger, M., "Simple models for complex nonequilibrium fluids," Phys. Rep. 390, 453-551 (2004).

Kröger, M., and S. Hess, "Rheological evidence for a dynamical crossover in polymer melts via nonequilibrium molecular dynamics," Phys. Rev. Lett. 85, 1128-1131 (2000).

Kröger, M., W. Loose, and S. Hess, "Structural changes and rheology of polymer melts via nonequilibrium molecular dynamics,” J. Rheol. 37, 1057-1079 (1993).

Laun, H. M., and H. Münstedt, "Elongational behavior of a low-density polyethylene melt," Rheol. Acta 17, 415-425 (1978).

Le, T. C., B. D. Todd, P. J. Daivis, and A. Uhlherr, "Structural properties of hyperbranched polymers in the melt under shear via nonequilibrium molecular dynamics simulation,” J. Chem. Phys. 130, 074901 (2009a).

Le, T. C., B. D. Todd, P. J. Daivis, and A. Uhlherr, "The effect of interbranch spacing on structural and rheological properties of hyperbranched polymer melts," J. Chem. Phys. 131, 044902 (2009b).

Likhtman, A. E., "Single-chain slip-link model of entangled polymers: Simultaneous description of neutron spin-echo, rheology, and diffusion," Mcromolecules 38, 6128-6139 (2005).

Likhtman, E., K. Sukumaran, and J. Ramirez, "Linear viscoelasticity from molecular dynamics simulation of entangled polymers,” Macromolecules 40, 6748-6757 (2007).

Matin, M. L., P. J. Daivis, and B. D. Todd, "Cell neighbour list method for planar elongational flow: Rheology of a diatomic fluid," Comput. Phys. Commun. 151, 35-46 (2003a). 
Matin, M. L., P. J. Daivis, and B. D. Todd, "Nonlinear shear and elongational rheology of polymer melts by non-equilibrium molecular dynamics,” J. Non-Newtonian Fluid Mech. 111, 1-18 (2003b).

McKinley, G. H., and T. Sridhar, "Filament-stretching rheometry of complex fluids," Annu. Rev. Fluid Mech. 34, 375-415 (2002).

McLeish, T. C. B., and R. G. Larson, "Molecular constitutive equations for a class of branched polymers: The pom-pom polymer," J. Rheol. 42, 81-110 (1998).

Neelov, I. M., and D. B. Adolf, "Brownian dynamics simulations of dendrimers under elongational flow: Beadrod model with hydrodynamic interactions," Macromolecules 36, 6914-6924 (2003).

Neelov, I. M., and D. B. Adolf, "Brownian dynamics simulations of hyperbranched polymers under elongational flow,” J. Phys. Chem. B 108, 7627-7636 (2004).

Nunez, C. M., Chiou, B.-S. Andrady, A. L., and Kahn, S. A., "Solution rheology of hyperbranched polyesters and their blends with linear polymers," Macromolecules 33, 1720-1726 (2000).

Petrie, C. J. S., "Extensional viscosity: A critical discussion,” J. Non-Newtonian Fluid Mech. 137, 15-23 (2006).

Rubinstein, M., and R. Colby, Polymer Physics (Oxford University Press, New York, 2003).

Sarkar, D., and M. Gupta, "Further investigation of the effect of elongational viscosity on entrance flow," J. Rein. Plast. Comp. 20, 1473-1484 (2001).

Silva, Jr., N. P., F. P. Menacho, and M. Chorilli, "Dendrimers as potential platform in nanotechnology based drug delivery systems," IOSR J. Pharm. 2, 23-30 (2012).

Sridhar, T., D. A. Nguyen, R. Prabhakar, and J. R. Prakash, "Rheological observation of glassy dynamics of dilute polymer solutions in elongational flows near the coil-stretch transition," Phys. Rev. Lett. 98, 167801 (2007).

Tanner, R. I., and S. Nasseri, "Simple constitutive models for linear and branched polymers," J. Non-Newtonian Fluid Mech. 116, 1-17 (2003).

Timoshenko, E. G., Y. A. Kuznetsov, and R. Connolly, "Conformations of dendrimers in dilute solution," J. Chem. Phys. 117, 9050 (2002).

Todd, B. D., and P. J. Daivis, "Nonequilibrium molecular dynamics simulations of planar elongational flow with spatially and temporally periodic boundary conditions," Phys. Rev. Lett. 81, 1118-1121 (1998).

Todd, B. D., and P. J. Daivis, “The stability of nonequilibrium molecular dynamics simulations of elongational flows," J. Chem. Phys. 112, 40-46 (2000).

Todd, B. D., and P. J. Daivis, "Homogeneous nonequilibrium molecular dynamics simulations of viscous flow: Techniques and applications," Mol. Simul. 33, 189-229 (2007).

Tomalia, D. A., J. R. Dewald, M. R. Hall, S. J. Martin, and P. B. Smith, "Discovery of dendrimers and dendritic polymers: A brief historical perspective," in Preprints of the 1st SPSJ International Polymer Conference (Society of Polymer Conference, Society of Polymer Science Japan, Kyoto, 1984), p. 65.

van Ruymbeke, E., C. Bailly, R. Keunings, and D. Vlassopoulos, "A general methodology to predict the linear rheology of branched polymers," Macromolecules 39, 6248-6259 (2006).

van Ruymbeke, E., K. Orfanou, H. Iatrou, M. Pitsikalis, N. Hadjichristidis, D. J. Lohse, and D. Vlassopoulos, "Entangled dendritic polymers and beyond: Rheology of symmetric Cayley-tree polymers and macromolecular self-assemblies," Macromolecules 40, 5941-5952 (2007).

Wagner, H., and H. Rolon-Garrido, "Nonlinear rheology of linear polymer melts: Modeling chain stretch by interchain tube pressure and Rouse time,” Korea-Australia Rheol. J. 21, 203-211 (2009).

Weinbergert, C. B., and J. D. Goddard, "Extensional flow behavior of polymer solutions and particle suspensions in a spinning motion,” Int. J. Multiphase Flow 1, 465-486 (1974).

Wooley, K. L., C. J. Hawker, and J. M. J. Frechet, "Hyperbranched macromolecules via a novel double-stage convergent growth approach,” J. Am. Chem. Soc. 113, 4252-4261 (1991). 